\title{
QUEEN'S UNIVERSITY
}

\section{POLYMERS RESEARCH GROUP}

\author{
19 Division Street, Kingston, ON, K7L 3N6 Canada
}

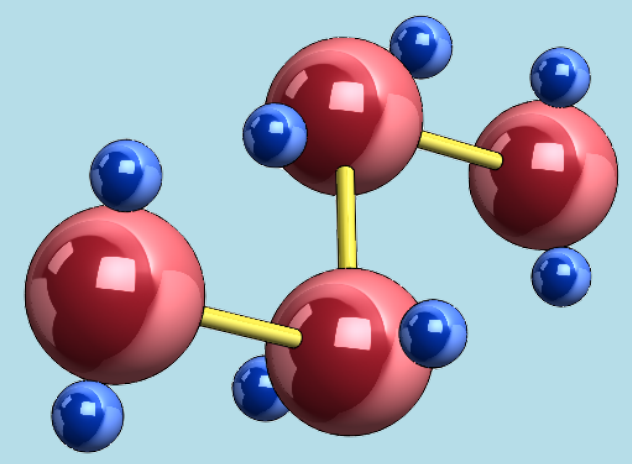

\section{KNUCKLE FORMATION FROM MELT ELASTICITY IN PLASTIC PIPE EXTRUSION}

\author{
C. Saengow ${ }^{1,3}$, A.J. Giacomin ${ }^{1,2, *}$ and C. Kolitawong ${ }^{3}$ \\ ${ }^{1}$ Polymers Research Group, \\ Chemical Engineering Department \\ ${ }^{2}$ Mechanical and Materials Engineering Department \\ Queen's University, Kingston, \\ Ontario, CANADA K7L 3N6 \\ ${ }^{3}$ Polymer Research Center \\ Mechanical and Aerospace Engineering Department \\ King Mongkut's University of Technology North Bangkok \\ Bangkok, THAILAND 10800
}

This report is circulated to persons believed to have an active interest in the subject matter; it is intended to furnish rapid communication and to stimulate comment, including corrections of possible errors.

*Corresponding author (giacomin@queensu.ca) 


\title{
KNUCKLE FORMATION FROM MELT ELASTICITY IN PLASTIC PIPE EXTRUSION
}

\author{
C. Saengow ${ }^{1,3}$, A.J. Giacomin ${ }^{1,2, *}$ and C. Kolitawong ${ }^{3}$ \\ ${ }^{1}$ Polymers Research Group, \\ Chemical Engineering Department \\ ${ }^{2}$ Mechanical and Materials Engineering Department \\ Queen's University, Kingston, \\ Ontario, CANADA K7L 3N6 \\ ${ }^{3}$ Polymer Research Center \\ Mechanical and Aerospace Engineering Department \\ King Mongkut's University of Technology North Bangkok \\ Bangkok, THAILAND 10800
}

\begin{abstract}
When plastic pipe is solidified, it proceeds through a long cooling chamber. Inside the chamber, inside the extrudate, the plastic is molten, and this inner surface solidifies last. Sag then happens inside this cooling chamber, and sometimes, thickened regions (called knuckles) arise in the lower quadrants of the pipe, and specifically in large diameter thick-walled pipes. To compensate for sag, we normally shift the die centerpiece downward. Here, we investigate this die eccentricity, and its remarkable interaction with fluid elasticity in knuckle formation in annular flow of polymeric liquids.

We develop a map to help plastics engineers predict the extrudate shape, including extrudate knuckles, and then from the mass balance over the postdie region, to predict the extrudate shape entering the cooling chamber. We find that Newtonian extrudates, or extrudates for small pipe, never knuckle. Both of these findings agree with industrial experience. We also include a worked example to help plastics engineers suppress extrudate knuckling.
\end{abstract}

Keywords: knuckle formation; slumping, sag, knuckling; melt fracture; Oldroyd 8-constant fluid; extrusion process.

${ }^{*}$ Corresponding author: giacomin@queensu.ca 


\section{CONTENTS}

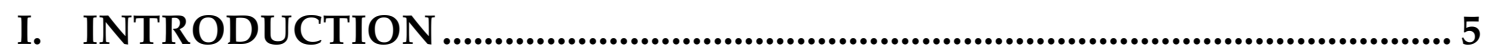

a. Eccentric Cylindrical Coordinates ............................................................... 7

b. Oldroyd 8-Constant Model ..................................................................... 9

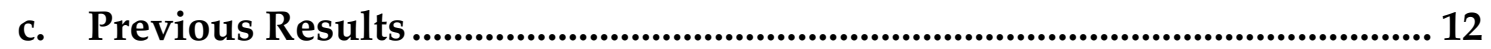

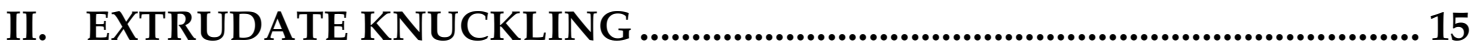

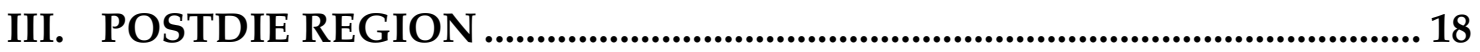

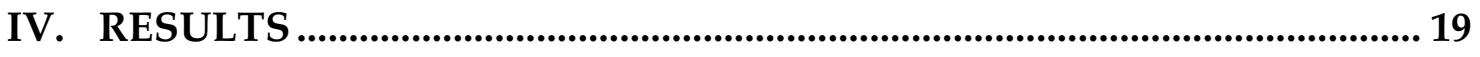

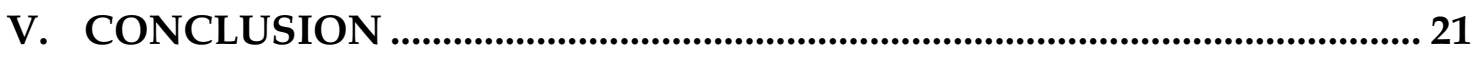

VI. WORKED EXAMPLE ................................................................................. 21

VII. ACKNOWLEDGMENT ...................................................................... 22

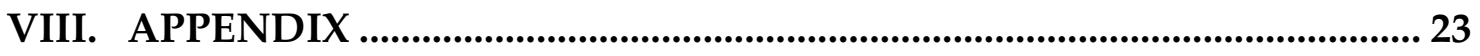

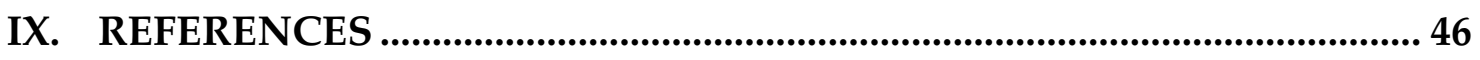




\section{TABLES}

Table 1: Dimensional Variables ......................................................................... 25

Table 2: Dimensionless Variables and Groups ...................................................... 27

Table 3: Solid pipe thickness profile (see APPENDIX E of [5]) ............................ 29

\section{FIGURES}

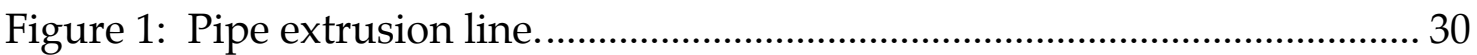

Figure 2: End view of pipe extrusion die defining the die dimensions............. 31

Figure 3: Side view of pipe extrusion die (left), defining the die dimensions, and the postdie region (right). The mandrel and barrel surfaces correspond to $\xi=\xi_{i}$ and $\xi=\xi_{o}$

Figure 4: $\xi_{i}$ and $\xi_{o}$ versus aspect ratio, $\kappa \equiv R_{i} / R_{o}$ with curves of constant $\epsilon \ldots 33$

Figure 5: Knuckling from sag (slump). $R_{i}, R_{o}, \delta, \Delta$ and $\Delta_{\mathrm{p}}$ are inner and outer radii, dimensional eccentricity, the thickness of the extrudate that leaving the die, and entering the cooling chamber.......................................... 34

Figure 6: Sag without knuckling........................................................................... 35

Figure 7: The shifted Cartesian coordinates, $(\tilde{x}, \tilde{y})$, versus the Cartesian

coordinates in Figure 8, $(x, y)$. The origin of the cylindrical coordinates, $(r, \tilde{\theta})$, is located at $(x, y)=\left(a \xi /\left[1-\xi^{2}\right], 0\right)$.

Figure 8: Eccentric cylindrical coordinates showing the circles of constant eccentric radial coordinate (black), $\xi$, and the circles of constant eccentric angular coordinate (green), $\theta$. Cyan example between $\xi=0.2$ and $\xi=0.4$.

Figure 9: Differential volume element of the molten plastic in eccentric cylindrical coordinates (cyan). Edges of differential surface element through which melt flows labeled $\left[a /\left(1-2 \xi \cos \theta+\xi^{2}\right)\right] d \xi$ and $\left[a /\left(1-2 \xi \cos \theta+\xi^{2}\right)\right] d \theta$. Cylindrical shell of constant eccentric cylindrical radial coordinate $\xi$ parallels $z$-axis, and intersects $x$-axis at $x=a /\left(1-\xi^{2}\right)$.

Figure 10: Measured wall thickness distribution of pipe with knuckles (red) with ten specific angular positions, for both cylindrical, $\tilde{\theta}$, and eccentric cylindrical, $\theta$, coordinates [Table 3]. Black circles represent the same eccentric die $\left(\xi_{i}, \xi_{o}\right)=(0.0592,0.0740)$ of Figure 16 . 
Figure 11: Newtonian contribution to the extrudate shape gradient. Four sets of increasing parametrized curves of $\xi_{i}$ from top to bottom [Eqs. (55)]. $\xi_{o}$ is increasing from bottom to top.

Figure 12: Non-Newtonian contribution to extrudate shape gradient. Four sets of increasing parametrized curves of $\xi_{i}$ (top to bottom). $\xi_{o}$ is increasing from bottom to top. Dashed curves are for the negative part of $-\partial\langle\breve{v}\rangle_{\theta, 1} / \partial \theta$ [Eqs. (56)]

Figure 13: Knuckling suppression map of the critical values of $(1-\sigma) S, \mathbb{K}$, versus inner contour, $\xi_{i}$, parametrized with $\xi_{o}$ [Eq. (59)]. Black dot illustrates the critical value for Figure 14.

Figure 14: Suppressing extrudate knuckles. Blue curves are the Newtonian (negative) contribution to extrudate shape gradient [Eq. (55)], and the green ones, non-Newtonian [Eq. (56)], using $\left(\xi_{i}, \xi_{0}\right)=(0.01,0.02)$. Critical value is $\mathbb{K}=0.00263$. Suppressing by decreasing $(1-\sigma) S$. Dashed curves are for the negative part of $-\partial\langle\breve{v}\rangle_{\theta, 1} / \partial \theta$

Figure 15: Average velocity of each slice $\theta$ versus eccentric angular position, $\theta$, with curves of constant $(1-\sigma) S=0.00557,0.02,0.04,0.06,0.08$ [Eq. (59)].

The smoothest curve, $(1-\sigma) S=\mathbb{K}=0.00557$, represents the critical extrudate knuckling 44

Figure 16: Thickness distribution of the extrudate entering the cooling chamber, $\Delta_{\mathrm{p}}=R_{o \mathrm{p}}-R_{\text {ip }}$, (blue) predicted from Eq. (67) [with Eq. (47)] using $P=8.46 \times 10^{5} \mathrm{~Pa} / \mathrm{m}, \eta_{0}=9.6 \mathrm{MPa} \cdot \mathrm{s}, V=1.98 \times 10^{-5} \mathrm{~m} / \mathrm{s}, R_{i}=0.351 \mathrm{~m}$, $R_{o}=0.44 \mathrm{~m}, R_{\text {op }}=0.44 \mathrm{~m}, \delta=0.0118 \mathrm{~m}, \lambda_{1}=2.38 \mathrm{~s}$ ， $\lambda_{2}=\mu_{0}=\mu_{1}=\mu_{2}=v_{1}=v_{2}=0$ versus the die shape (red). 


\section{INTRODUCTION}

After plastic pipe extrudate emerges from the annular die, it proceeds through a long cooling chamber (see Figure 1) and is solidified by either bathing or spraying the outer surface or the inner with cold water. Inside this cooling chamber, inside the extrudate, the plastic is still molten, and the inner surface solidifies last. A gravitational flow thus happens in the cooling chamber, and we call this sag $[1,2,3,4,5,6,7]$.

To compensate for sag, we normally shift the die centerpiece downward. Here, we investigate this die eccentricity, and its remarkable interaction with fluid elasticity in knuckle formation in annular flow of polymeric liquids. To compensate for sag, engineers also rotate the pipe gently [2,7].

By extrudate, we mean the molten plastic that just emerged from the extrusion die, and the term pipe refers to the completely solidified extrudate. Curiously, sometimes the pipe is thickest, not at the bottom, but elsewhere in its lower quadrants (see Figure 5; Fig. 1 and Fig. 6 of [6]). By lower quadrants, we mean, in cylindrical coordinates, where $\pi / 2 \leq \tilde{\theta} \leq 3 \pi / 2$ (see Figure 7). These thickened sections in the lower pipe quadrants are called knuckles, and their formation, knuckling (or slump $[1,4,5,6]$ ). Knuckling must not to be confused with sag (compare Figure 5 with Figure 6; also [8]). Whereas sag can reshape knuckled extrudate, sag can also itself knuckle extrudate that enters the cooling chamber without knuckles $[1,4,5,6]$. To be clear, extrudate knuckling refers to the molten extrudate, and not to the pipe.

Pipe knuckling has been attributed to sag of eccentric extrudates $[1,4,5,6]$. By pipe knuckling, we mean when completely solidified product has thickened 
sections in its lower quadrants. Although numerical simulation of sag of eccentric extrudates does qualitatively predict some knuckling, observed knuckling is normally far more pronounced (see Fig. 6 of [6]). Specifically, profiles predicted by sag alone over-predict the bottom thickness. Pipe knuckling is thus one of the greatest mysteries of plastic pipe manufacture.

Pipe knuckling has been observed on extrusion lines producing large diameter thick-walled pipes when the extrusion die is eccentric $[1,5,6]$. Figure 10 illustrates the detailed example of pipe knuckling in large thick high-density polyethylene pipe. When pipe knuckles arise, they can be a serious nonuniformity of thickness. Moreover, when large diameter pipe is made, from a new resin, it can take up to four attempts at start-up to get an acceptable thickness profile [9]; each attempt can waste up to $10 \mathrm{~h}$ and ten thousand pounds of pipe resin $[10,11]$. Pipe knuckling generally worsens start-up, often to the point of no solution, and thus, to de-selection of otherwise desirable new pipe resins.

By pipe or extrudate thickness, we mean the radial distance measured from the outer shell to the inner shell along the radial cylindrical coordinate, centered on the outer cylinder (see Figure 10). Eq. (80) [Eq. (81)] relates the angular positions in cylindrical coordinates, $\tilde{\theta}$, to the angular position in eccentric cylindrical coordinates, $\theta$ [vice versa]. These relations can be used to transform a thickness distribution from one coordinate system to another.

Fluid elasticity plays an important role in plastic pipe extrusion. It governs the characteristic of the plastic pipe: (i) throughput, (ii) residual stress, (iii) rigidity of the mandrel attachment and (iv) the extrudate shape (see $\S 5.2$ of 
[12]). In this paper, we undertake a detailed examination of out previous result for the extrudate shape, and discover extrudate knuckling, and thus a new explanation for pipe knuckling.

In this paper, we find that extrudate knuckling is governed by (i) melt elasticity, (ii) pressure gradient across the die and (iii) die geometry including especially its eccentricity (without which, we find that, no extrudate knuckling can arise, see Fig. 3 of [6]). We develop a map to help plastics engineers predict the extrudate shape, including extrudate knuckles, and then from the mass balance, to predict the final pipe shape. We also include a worked example to help plastics engineers control pipe shape, by suppressing extrudate knuckling.

\section{a. Eccentric Cylindrical Coordinates}

Eccentric cylindrical coordinates, illustrated in Figure 8, consist of two orthogonal sets of cylindrical shells. The first set is defined by the eccentric radial coordinate, $\xi$, and another, by the eccentric angular coordinate, $\theta$. The transformations from Cartesian to eccentric cylindrical coordinates are given by:

$$
\begin{aligned}
& x=a \frac{1-\xi \cos \theta}{1-2 \xi \cos \theta+\xi^{2}} \\
& y=a \frac{\xi \sin \theta}{1-2 \xi \cos \theta+\xi^{2}} \\
& z_{\mathrm{C}}=z
\end{aligned}
$$

or:

$$
\xi=\frac{x^{2}+y^{2}-2 a x+a^{2}}{x^{2}+y^{2}}
$$


$\theta=\arctan \left(\frac{a y}{x^{2}+y^{2}-a x}\right)$

$z=z_{C}$

where the $z$-coordinate is the dimensional flow direction (see Figure 3), and we call:

$a \equiv \delta^{-1}\left(R_{o}^{4}+R_{i}^{4}+\delta^{4}-2 R_{i}^{2} R_{o}^{2}-2 R_{i}^{2} \delta^{2}-2 R_{o}^{2} \delta^{2}\right)^{1 / 2}$

the dimensional confocal length, and thus we define the dimensionless confocal length as:

$f \equiv \frac{a}{R_{o}} \equiv \epsilon^{-1}\left(1+\kappa^{4}+\epsilon^{4}-2 \kappa^{2}-2 \kappa^{2} \epsilon^{2}-2 \epsilon^{2}\right)^{1 / 2}$

where $R_{i}, R_{o}$ and $\delta$ are the mandrel radius, the extrusion die radius and the dimension eccentricity (see Figure 2 and Figure 3 for variable definitions). For convenience, we define every dimensional symbol in Table 1, and dimensionless ones in Table 2.

Figure 8 shows the circular cross-sections of cylindrical shells of constant $\xi$ (black circles) and of constant $\theta$ (green circles). Eccentric cylindrical coordinates are not to be confused with bipolar cylindrical coordinates, which are also used for analyzing flow in eccentric annuli (see FIGURE A.7-1. In [13] or in [14], or Fig. 3. In [15], or Fig. P3.16 in [16] where $\xi$ and $\theta$ have other meanings; $[17,18,19,20,21,22])$.

We next illustrate our eccentric cylindrical differential volume element in Figure 9. Employing Eqs. (A.7-13) of [23] gives us the scale factors for coordinates $\xi$ and $\theta$ : 


$$
\begin{aligned}
& h_{\xi}=\frac{a}{1+\xi^{2}-2 \xi \cos \theta} \\
& h_{\theta}=\frac{a \xi}{1+\xi^{2}-2 \xi \cos \theta} \\
& h_{z}=1
\end{aligned}
$$

The differential area relevant to the axial flow rate is given by:

$$
d A_{\xi \theta}=\frac{a^{2} \xi}{\left(1+\xi^{2}-2 \xi \cos \theta\right)^{2}} d \xi d \theta
$$

We will use Eq. (12) to get the axial flow rate, and also the extrudate shape in Section II.

\section{b. Oldroyd 8-Constant Model}

In this paper, we choose the Oldroyd 8-constant constitutive model, Eq. (13), for the extruded plastics. We choose this model for its rich diversity of special cases. Eq. (13) has been treated in some detail in (see Chapters 2 and 3 in [24]; $\S 8.1$ of [13]; §7.3 in [14]; Section 4.2. in [25]; Section 6.3-2 in [26]; Section 6.1-7 in [27] and Section 3.4.5 in [28]). Eq. (13) can be written in integral form (see EXAMPLE 8.1-2 of [13]). Tables 8.1-1 in [13] or 7.3-2 in [14] identify eleven special cases of Eq. (13) (see Tables 8.1-8.3 in [29]). Additionally, the Oldroyd 8-constant framework has been closely connected, albeit approximately, with macromolecular theory ([30,31,32]; see Table 1 of [33]; see Eqs. (32) of [34],[35]; see Tables 6.2-1 and 6.2-2 of [14]; Problems 11B.9 and 11B.10 of [36]; §IV and §V. of [37]; §9.5 of [13]).

We write the Oldroyd 8-constant model as: 


$$
\begin{aligned}
\tau_{i k} & +\lambda_{1} \frac{\mathscr{O} \tau_{i k}}{\mathscr{O} t}+\mu_{0} \tau_{j j} \dot{\gamma}_{i k}-\mu_{1}\left(\tau_{i j} \dot{\gamma}_{i k}+\tau_{j k} \dot{\gamma}_{i j}\right)+v_{1} \tau_{j l} \dot{\gamma}_{j l} \delta_{i k} \\
& =2 \eta_{0}\left(\dot{\gamma}_{i k}+\lambda_{2} \frac{\mathscr{O} \dot{\gamma}_{i k}}{\mathscr{O} t}-2 \mu_{2} \dot{\gamma}_{i j} \dot{\gamma}_{j k}+v_{2} \dot{\gamma}_{i} \dot{\gamma}_{j l} \delta_{i k}\right)
\end{aligned}
$$

where:

$$
\pi_{i j} \equiv \tau_{i k}+p \delta_{i k}
$$

and the rate-of-strain tensor is given by:

$\dot{\gamma}_{i k} \equiv \frac{1}{2}\left(\frac{\partial v_{k}}{\partial x_{i}}+\frac{\partial v_{i}}{\partial x_{k}}\right)$

and the corotational derivative, by:

$\frac{\mathscr{O} b_{i k}}{\mathscr{O} t} \equiv \frac{\partial b_{i k}}{\partial t}+v_{j} \frac{\partial b_{i k}}{\partial x_{i}}+\omega_{i j} b_{j k}+\omega_{k j} b_{i j}$

where the $i j$-component of the vorticity tensor is given by:

$\omega_{i j} \equiv \frac{1}{2}\left(\frac{\partial v_{k}}{\partial x_{i}}-\frac{\partial v_{i}}{\partial x_{k}}\right)$

In Eq. (14), $\delta_{i k}$ is the kronecker delta.

For steady shear flow, the viscometric functions are given by:

$$
\frac{\eta(\dot{\gamma})}{\eta_{0}}=-\frac{\Psi_{1}(\dot{\gamma})}{\Psi_{10}}\left(\frac{\lambda_{2}}{\lambda_{1}}-1\right)+\frac{\lambda_{2}}{\lambda_{1}}=\frac{\Psi_{2}(\dot{\gamma})}{\Psi_{20}}\left(\frac{\lambda_{1}-\lambda_{2}-\mu_{1}+\mu_{2}}{\lambda_{1}-\mu_{1}}\right)+\frac{\lambda_{2}-\mu_{2}}{\lambda_{1}-\mu_{1}}=\frac{1+\sigma_{2} \dot{\gamma}^{2}}{1+\sigma_{1} \dot{\gamma}^{2}}
$$

where:

$$
\begin{aligned}
& \Psi_{10} \equiv 2 \eta_{0}\left(\lambda_{1}-\lambda_{2}\right) \\
& \Psi_{20} \equiv-\eta_{0}\left(\lambda_{1}-\lambda_{2}-\mu_{1}+\mu_{2}\right)
\end{aligned}
$$

and where:

$$
\sigma_{1} \equiv \lambda_{1}^{2}+\mu_{0}\left(\mu_{1}-\frac{3}{2} v_{1}\right)-\mu_{1}\left(\mu_{1}-v_{1}\right)
$$


$\sigma_{2} \equiv \lambda_{1} \lambda_{2}+\mu_{0}\left(\mu_{2}-\frac{3}{2} v_{2}\right)-\mu_{1}\left(\mu_{2}-v_{2}\right)$

and where the magnitude of the rate of deformation tensor:

$\dot{\gamma} \equiv \frac{\left(1-2 \xi \cos \theta+\xi^{2}\right)}{a \xi} \sqrt{\left(\xi \frac{\partial v_{z}}{\partial \xi}\right)^{2}+\left(\frac{\partial v_{z}}{\partial \theta}\right)^{2}}$

is obtained by substituting Eqs. (33a) of [38] and (33b) of [38] into Eq. (43) of [38]. Introducing the Weissenberg number:

$\mathrm{Wi} \equiv \sqrt{\sigma_{1}} \dot{\gamma}$

into Eq. (18) gives:

$\frac{\eta}{\eta_{0}}=-\frac{\Psi_{1}}{\Psi_{10}}\left(\frac{\lambda_{2}}{\lambda_{1}}-1\right)+\frac{\lambda_{2}}{\lambda_{1}}=\frac{\Psi_{2}}{\Psi_{20}}\left(\frac{\lambda_{1}-\lambda_{2}-\mu_{1}+\mu_{2}}{\lambda_{1}-\mu_{1}}\right)+\frac{\lambda_{2}-\mu_{2}}{\lambda_{1}-\mu_{1}}=\frac{1+\sigma \mathrm{Wi}^{2}}{1+\mathrm{Wi}^{2}}$

where:

$\sigma \equiv \sigma_{2} / \sigma_{1}$

Taking the limit as strain rate, $\dot{\gamma}$, goes to infinity yields:

$\eta_{\infty} / \eta_{0}=\sigma$

From Eq. (25) we learn that, for the shear stress to increase monotonically with shear rate:

$\sigma \geq 1 / 9$

The viscosity curves will inflect at (see Eq. (90) of [39]):

$\left(\mathrm{Wi}_{\mathrm{i}}, \eta_{i}\right)=\left(1 / \sigma^{1 / 4}, \sqrt{\sigma}\right)$

By viscosity curves we mean curves of $\ln \eta$ versus $\ln \mathrm{Wi}$. Further:

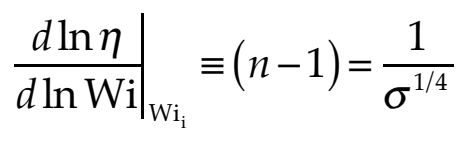

where $n$ is called the power-law index. 
The Oldroyd 8-constant model describes shear thinning $(\sigma<1)$, and shear thickening $(\sigma>1)$, and the behaviors of the constant viscosity fluids $(\sigma=1)$. In plastic pipe extrusion, we find example of shear thinning (polyolefins) [40], shear thickening (short glass fiber filled polyolefins) [41], and constant viscosity fluids (tubing from condensation polymers such as nylon). Using this model thus allows us to deepen our understanding of this wide variety of pipe extrusion problems.

\section{c. Previous Results}

The dimensionless axial velocity profile is given by [12]:

$\breve{v}_{z} \equiv 4 \eta_{0} v_{z} / a^{2} P=v_{0}+(1-\sigma) S v_{1}$

where the Newtonian contribution is given by:

$$
v_{0}=\sum_{n=0}^{\infty} \phi_{n} \cos n \theta+\frac{-1}{1-2 \xi \cos \theta+\xi^{2}}
$$

where:

$\phi_{n}=\left\{\begin{array}{cc}\alpha_{0}+\beta_{0} \ln \xi^{2} & ; n=0 \\ 2 \alpha_{n} \xi^{n}+2 \beta_{n} \xi^{-n} & ; n \geq 1\end{array}\right.$

with $\alpha_{n}, \beta_{n}$ which are given by:

$\alpha_{n}= \begin{cases}\frac{\xi_{i}^{2} \ln \xi_{i}^{2}+\ln \xi_{o}^{2}-\xi_{o}^{2} \ln \xi_{o}^{2}-\ln \xi_{i}^{2}}{\left(-\ln \xi_{o}^{2}+\xi_{o}^{2} \ln \xi_{o}^{2}+\ln \xi_{i}^{2}-\xi_{o}^{2} \ln \xi_{i}^{2}\right)\left(-1+\xi_{i}^{2}\right)} & ; n=0 \\ \frac{\xi_{i}^{2} \xi_{o}^{2 n}-\xi_{o}^{2 n}+\xi_{i}^{2 n}-\xi_{o}^{2} \xi_{i}^{2 n}}{\left(\xi_{o}^{2 n}-\xi_{o}^{2 n+2}-\xi_{i}^{2 n}+\xi_{o}^{2} \xi_{i}^{2 n}\right)\left(-1+\xi_{i}^{2}\right)} & ; n \geq 1\end{cases}$ 


$$
\beta_{n}=\left\{\begin{array}{cl}
\frac{\xi_{o}^{2}-\xi_{i}^{2}}{\left(-\ln \xi_{o}^{2}+\xi_{o}^{2} \ln \xi_{o}^{2}+\ln \xi_{i}^{2}-\xi_{o}^{2} \ln \xi_{i}^{2}\right)\left(-1+\xi_{i}^{2}\right)} & ; n=0 \\
\frac{-\xi_{i}^{n} \xi_{o}^{n}\left(\xi_{o}^{2}-\xi_{i}^{2}\right)}{\left(-\xi_{o}^{n} \xi_{i}^{-n}+\xi_{o}^{n+2} \xi_{i}^{-n}+\xi_{o}^{-n} \xi_{i}^{n}-\xi_{o}^{-n+2} \xi_{i}^{n}\right)\left(-1+\xi_{i}^{2}\right)} & ; n \geq 1
\end{array}\right.
$$

and $\xi_{i}$ and $\xi_{o}$, by:

$$
\begin{aligned}
& \xi_{i} \equiv \frac{\left(R_{o}^{2}-R_{i}^{2}-\delta^{2}\right)-\left(R_{o}^{4}+R_{i}^{4}+\delta^{4}-2 R_{i}^{2} R_{o}^{2}-2 R_{i}^{2} \delta^{2}-2 R_{o}^{2} \delta^{2}\right)^{1 / 2}}{2 \delta R_{i}} ; \delta<\left(R_{o}-R_{i}\right) \\
& \xi_{o} \equiv \frac{\left(R_{o}^{2}-R_{i}^{2}+\delta^{2}\right)-\left(R_{o}^{4}+R_{i}^{4}+\delta^{4}-2 R_{i}^{2} R_{o}^{2}-2 R_{i}^{2} \delta^{2}-2 R_{o}^{2} \delta^{2}\right)^{1 / 2}}{2 \delta R_{o}} ; \delta<\left(R_{o}-R_{i}\right)
\end{aligned}
$$

or in dimensionless terms:

$$
\begin{aligned}
& \xi_{i} \equiv \frac{\left(1-\kappa^{2}-\epsilon^{2}\right)-\left(1+\kappa^{4}+\epsilon^{4}-2 \kappa^{2}-2 \kappa^{2} \epsilon^{2}-2 \epsilon^{2}\right)^{1 / 2}}{2 \epsilon \kappa} ; \epsilon<(1-\kappa) \\
& \xi_{o} \equiv \frac{\left(1-\kappa^{2}+\epsilon^{2}\right)-\left(1+\kappa^{4}+\epsilon^{4}-2 \kappa^{2}-2 \kappa^{2} \epsilon^{2}-2 \epsilon^{2}\right)^{1 / 2}}{2 \epsilon} ; \epsilon<(1-\kappa)
\end{aligned}
$$

We use Eqs. (38) and (39) to plot Figure 4. The non-Newtonian variable by:

$$
v_{1}=\sum_{n=0}^{\infty} \psi_{n} \cos n \theta
$$

which can be truncated to:

$$
v_{1}=\sum_{n=0}^{5} \psi_{n} \cos n \theta
$$

where:

$\psi_{n}=\beta_{0}^{3} \psi_{n}^{(1)}+\beta_{0}^{2} \psi_{n}^{(2)}+\beta_{0} \psi_{n}^{(3)}+\psi_{n}^{(4)}$

with: 
$\psi_{n}^{(m)}=\left\{\begin{array}{lll}\mathrm{A}_{0}^{(m)}+\mathrm{B}_{0}^{(m)} \ln \xi^{2}+v_{1 p, 0}^{(m)} & ; n=0 & ; m=1,2,3,4 \\ \mathrm{~A}_{n}^{(m)} \xi^{n}+\mathrm{B}_{n}^{(m)} \xi^{-n}+v_{1 p, n}^{(m)} & ; n \geq 1 & ; m=1,2,3,4\end{array}\right.$

where $v_{1 p, n}^{(m)}$ are given by Eqs. (82) - (100) of [12], and where $A_{n}^{(m)}$ and $B_{n}^{(m)}$ are given by:

$$
\begin{aligned}
& A_{n}^{(m)}= \begin{cases}\frac{\ln \xi_{o}^{2} v_{1 p, 0}^{(m)}\left(\xi_{i}\right)-\ln \xi_{i}^{2} v_{1 p, 0}^{(m)}\left(\xi_{o}\right)}{\ln \xi_{i}^{2}-\ln \xi_{o}^{2}} & ; n=0 \\
\frac{\xi_{i}^{-n} v_{1 p, n}^{(m)}\left(\xi_{o}\right)-\xi_{o}^{-n} v_{1 p, n}^{(m)}\left(\xi_{i}\right)}{\xi_{o}^{-n} \xi_{i}^{n}-\xi_{o}^{n} \xi_{i}^{-n}} & ; n \geq 1\end{cases} \\
& B_{n}^{(m)}= \begin{cases}\frac{v_{1 p, 0}^{(m)}\left(\xi_{i}\right)-v_{1 p, 0}^{(m)}\left(\xi_{o}\right)}{\ln \xi_{o}^{2}-\ln \xi_{i}^{2}} & ; n=0 \\
\frac{\xi_{o}{ }^{n} v_{1 p, n}^{(m)}\left(\xi_{i}\right)-\xi_{i}^{n} v_{1 p, n}^{(m)}\left(\xi_{o}\right)}{\xi_{o}^{-n} \xi_{i}^{n}-\xi_{o}{ }^{-n}} & ; n \geq 1\end{cases}
\end{aligned}
$$

Substituting Eqs. (32) and (41) into Eq. (31) gives:

$$
\breve{v}_{z}=\sum_{n=0}^{\infty} \phi_{n} \cos n \theta+\frac{-1}{1-2 \xi \cos \theta+\xi^{2}}+(1-\sigma) S \sum_{n=0}^{5} \psi_{n} \cos n \theta
$$

Recalling that (see Eq. (108), (112) and (113) of [12]):

$$
\left\langle\breve{v}_{z}\right\rangle_{\theta}=\left\langle\breve{v}_{z}\right\rangle_{\theta, 0}+(1-\sigma) S\left\langle\breve{v}_{z}\right\rangle_{\theta, 1}
$$

where:

$$
\begin{aligned}
& \left\langle\breve{v}_{z}\right\rangle_{\theta, 0} \equiv \frac{f^{2}}{\pi\left(1-\kappa^{2}\right)} \int_{\xi_{i}}^{\xi_{\xi_{0}}} \frac{\xi}{\left(1+\xi^{2}-2 \xi \cos \theta\right)^{2}} v_{0} d \xi \\
& \left\langle\breve{v}_{z}\right\rangle_{\theta, 1} \equiv \frac{f^{2}}{\pi\left(1-\kappa^{2}\right)} \int_{\xi_{i}}^{\xi_{0}} \frac{\xi}{\left(1+\xi^{2}-2 \xi \cos \theta\right)^{2}} v_{1} d \xi
\end{aligned}
$$

which we will use below. 


\section{EXTRUDATE KNUCKLING}

In this section, we use Eq. (47) from our previous results to investigate, and to eventually explain, extrudate knuckling. We develop a map to help plastics engineers predict extrudate shape, including extrudate knuckles, and then, from the mass balance, to predict the pipe shape (in Section III).

To test for the presence of extrudate knuckles, we begin by differentiating Eq. (47) once:

$\frac{\partial\left\langle\breve{v}_{z}\right\rangle_{\theta}}{\partial \theta}=\frac{\partial\left\langle\breve{v}_{z}\right\rangle_{\theta, 0}}{\partial \theta}+(1-\sigma) S \frac{\partial\left\langle\breve{v}_{z}\right\rangle_{\theta, 1}}{\partial \theta}$

and twice to give:

$\frac{\partial^{2}\left\langle\breve{v}_{z}\right\rangle_{\theta}}{\partial \theta^{2}}=\frac{\partial^{2}\left\langle\breve{v}_{z}\right\rangle_{\theta, 0}}{\partial \theta^{2}}+(1-\sigma) S \frac{\partial^{2}\left\langle\breve{v}_{z}\right\rangle_{\theta, 1}}{\partial \theta^{2}}$

Setting the left side of Eq. (50) to zero gives:

$\frac{\partial\left\langle\breve{v}_{z}\right\rangle_{\theta, 0}}{\partial \theta}+(1-\sigma) S \frac{\partial\left\langle\breve{v}_{z}\right\rangle_{\theta, 1}}{\partial \theta}=0$

We will use this equation to solve for the angular position of the extrudate knuckles, $\theta_{\mathrm{K}}$. We will further identify under which process condition extrudate knuckles arise.

We next calculate both of the derivatives in Eq. (52). Substituting Eqs. (32) and (41) into Eqs. (48) and (49) gives:

$\left\langle\breve{v}_{z}\right\rangle_{\theta, 0}=\frac{f^{2}}{\pi\left(1-\kappa^{2}\right)} \int_{\xi_{i}}^{\xi_{0}} \frac{\xi\left[\sum_{n=0}^{\infty} \phi_{n} \cos n \theta+\frac{-1}{1-2 \xi \cos \theta+\xi^{2}}\right]}{\left(1+\xi^{2}-2 \xi \cos \theta\right)^{2}} d \xi$

and: 
$\left\langle\breve{v}_{z}\right\rangle_{\theta, 1}=\frac{f^{2}}{\pi\left(1-\kappa^{2}\right)} \int_{\xi_{i}}^{\xi_{0}} \frac{\xi \sum_{n=0}^{5} \psi_{n} \cos n \theta}{\left(1+\xi^{2}-2 \xi \cos \theta\right)^{2}} d \xi$

Differentiating Eqs. (53) and (54) once:

$$
\begin{aligned}
& \frac{\partial\left\langle\breve{v}_{z}\right\rangle_{\theta, 0}}{\partial \theta}=\frac{f^{2}}{\pi\left(1-\kappa^{2}\right)} \int_{\xi_{i}}^{\xi_{0}}\left[\begin{array}{c}
\frac{-\xi \sum_{n=1}^{\infty} n \phi_{n} \sin n \theta}{\left(1-2 \xi \cos \theta+\xi^{2}\right)^{2}}+\frac{-4 \xi^{2} \sin \theta \sum_{n=0}^{\infty} \phi_{n} \cos n \theta}{\left(1-2 \xi \cos \theta+\xi^{2}\right)^{3}} \\
+\frac{6 \xi^{2} \sin \theta}{\left(1-2 \xi \cos \theta+\xi^{2}\right)^{4}}
\end{array}\right] d \xi \\
& \frac{\partial\left\langle\breve{v}_{z}\right\rangle_{\theta, 1}}{\partial \theta}=\frac{f^{2}}{\pi\left(1-\kappa^{2}\right)} \int_{\xi_{i}}^{\xi_{\xi_{0}}}\left[\frac{-\xi \sum_{n=1}^{5} n \psi_{n} \sin n \theta}{\left(1-2 \xi \cos \theta+\xi^{2}\right)^{2}}+\frac{-4 \xi^{2} \sin \theta \sum_{n=0}^{5} \psi_{n} \cos n \theta}{\left(1-2 \xi \cos \theta+\xi^{2}\right)^{3}}\right] d \xi
\end{aligned}
$$

and twice gives:

$$
\begin{gathered}
\frac{\partial^{2}\left\langle\breve{v}_{z}\right\rangle_{\theta, 0}}{\partial \theta^{2}}=\frac{f^{2}}{\pi\left(1-\kappa^{2}\right)} \int_{\xi_{i_{i}}}^{\xi_{0}}\left[\begin{array}{l}
-\frac{\xi \sum_{n=1}^{\infty} n^{2} \phi_{n} \cos n \theta}{\left(1-2 \xi \cos \theta+\xi^{2}\right)^{2}}+\xi^{2} \frac{8 \sin \theta \sum_{n=1}^{\infty} n \phi_{n} \sin n \theta-4 \cos \theta \sum_{n=0}^{\infty} \phi_{n} \cos n \theta}{\left(1-2 \xi \cos \theta+\xi^{2}\right)^{3}} \\
+\frac{6 \xi^{2} \cos \theta+24 \xi^{3} \sin ^{2} \theta \sum_{n=0}^{\infty} \phi_{n} \cos n \theta}{\left(1-2 \xi \cos \theta+\xi^{2}\right)^{4}}-\frac{48 \xi^{3} \sin ^{2} \theta}{\left(1-2 \xi \cos \theta+\xi^{2}\right)^{5}}
\end{array}\right] d \xi \\
\frac{\partial^{2}\left\langle\breve{v}_{z}\right\rangle_{\theta, 1}}{\partial \theta^{2}}=\frac{f^{2}}{\pi\left(1-\kappa^{2}\right)} \int_{\xi_{i}}^{\xi_{0}}\left[\begin{array}{c}
-\frac{\xi \sum_{n=1}^{5} n^{2} \psi_{n} \cos n \theta}{\left(1-2 \xi \cos \theta+\xi^{2}\right)^{2}}+\frac{24 \xi^{3} \sin ^{2} \theta \sum_{n=1}^{5} \psi_{n} \cos n \theta}{\left(1-2 \xi \cos \theta+\xi^{2}\right)^{4}} \\
+\xi^{2} \frac{8 \sin \theta \sum_{n=1}^{5} n \psi_{n} \sin n \theta-4 \cos \theta \sum_{n=1}^{5} \psi_{n} \cos n \theta}{\left(1-2 \xi \cos \theta+\xi^{2}\right)^{3}}
\end{array}\right] d \xi
\end{gathered}
$$

Substituting both Eqs. (55) and (56) into Eq. (52) gives: 


$$
\begin{aligned}
& \int_{\xi_{i}}^{\xi_{o}}\left[\begin{array}{c}
\frac{-\xi \sum_{n=1}^{\infty} n \phi_{n} \sin n \theta_{\mathrm{K}}}{\left(1-2 \xi \cos \theta_{\mathrm{K}}+\xi^{2}\right)^{2}}+\frac{-4 \xi^{2} \sin \theta_{\mathrm{K}} \sum_{n=0}^{\infty} \phi_{n} \cos n \theta_{\mathrm{K}}}{\left(1-2 \xi \cos \theta_{\mathrm{K}}+\xi^{2}\right)^{3}} \\
+\frac{6 \xi^{2} \sin \theta_{\mathrm{K}}}{\left(1-2 \xi \cos \theta_{\mathrm{K}}+\xi^{2}\right)^{4}}
\end{array}\right] d \xi \\
& +(1-\sigma) S \int_{\xi_{i}}^{\xi_{o}}\left[\frac{-\xi \sum_{n=0}^{5} n \psi_{n} \sin n \theta_{\mathrm{K}}}{\left(1-2 \xi \cos \theta_{\mathrm{K}}+\xi^{2}\right)^{2}}+\frac{-4 \xi^{2} \sin \theta_{\mathrm{K}} \sum_{n=0}^{5} \psi_{n} \cos n \theta_{\mathrm{K}}}{\left(1-2 \xi \cos \theta_{\mathrm{K}}+\xi^{2}\right)^{3}}\right] d \xi=0
\end{aligned}
$$

When the solution to Eq. (59) also satisfies both:

$$
\frac{\partial^{2}\left\langle\breve{v}_{z}\right\rangle_{\theta, 0}}{\partial \theta^{2}}+(1-\sigma) S \frac{\partial^{2}\left\langle\breve{v}_{z}\right\rangle_{\theta, 1}}{\partial \theta^{2}}<0
$$

where $\partial^{2}\left\langle\breve{v}_{z}\right\rangle_{\theta, 0} / \partial \theta^{2}$ and $\partial^{2}\left\langle\breve{v}_{z}\right\rangle_{\theta, 1} / \partial \theta^{2}$ are given in Eqs. (57) and (58), and, in cylindrical coordinates:

$$
\frac{\pi}{2} \leq \tilde{\theta}_{\mathrm{K}}<\pi
$$

then the thickest parts of the extrudate are in the lower quadrants (see subhead of Section I above), and thus extrudate knuckles exist. Eq. (61) can be rewritten in eccentric cylindrical coordinates as:

$$
\arccos \left(\frac{2 \xi_{o}}{1+\xi_{0}^{2}}\right) \leq \theta_{\mathrm{K}}<\arccos \left(\frac{\left(1+\xi_{o}^{2}\right)\left(-\frac{R_{o}}{a}+\frac{1}{1-\xi_{o}^{2}}\right)-1}{2 \xi_{0}\left(-\frac{R_{o}}{a}+\frac{1}{1-\xi_{o}^{2}}\right)-\xi_{0}}\right)
$$

Eqs. (59), (60) and (61) [or Eq. (62)] are the criterion for extrudate knuckling. This criterion is the main result of this paper. 


\section{III.POSTDIE REGION}

In this section, we perform a mass balance on the postdie region. By postdie region, we mean the section of the manufacturing line between the die and the cooling chamber, where the annular melt undergoes extensional flow (see Figure 1 and Figure 3; $[42,43,44])$. Our mass balance on the postdie region gives the shape of the melt entering the cooling chamber as a function of the shape of the viscoelastic melt emerging from the eccentric annular die.

Assuming the melt density is constant in the postdie region, the mass balance at any particular $\theta$ is given by:

$$
\left\langle v_{z}\right\rangle_{\tilde{\theta}} d A_{\tilde{\theta}}=V d A_{\mathrm{p} \tilde{\theta}}
$$

which we then rearrange and, using Table 2, adimensionalize as:

$$
\frac{\left\langle v_{z}\right\rangle_{\tilde{\theta}}}{V}=\frac{d A_{\mathrm{p} \tilde{\theta}}}{d A_{\mathrm{e} \tilde{\theta}}}=\frac{\left\langle\breve{v}_{z}\right\rangle_{\tilde{\theta}}}{\widetilde{V}}
$$

The differential area for each slice of the extrudate is given by (see Fig. A.8-1. in [23]):

$d A_{\mathrm{p} \tilde{\theta}}=\int_{R_{\mathrm{ip}}(\tilde{\theta})}^{R_{o \mathrm{p}}} r d r=\frac{R_{o \mathrm{p}}^{2}-R_{i \mathrm{p}}^{2}(\tilde{\theta})}{2}$

and, for the pipe, by:

$d A_{\tilde{\theta}}=\frac{R_{o}^{2}-R_{i}^{2}(\tilde{\theta})}{2}$

Substituting Eqs. (65) and (66) into Eq. (64), and then solving for $R_{i p}(\tilde{\theta})$ gives:

$$
R_{i \mathrm{p}}(\tilde{\theta})=\sqrt{R_{o \mathrm{p}}^{2}-\mathrm{DDR}\left[R_{o}^{2}-R_{i}^{2}(\tilde{\theta})\right]}
$$

where the drawdown ratio is defined by:

$$
\mathrm{DDR} \equiv\left\langle\breve{v}_{z}\right\rangle_{\tilde{\theta}} / \breve{V}
$$


Eq. (67) is thus the main result of this section. We will use Eq. (67) to convert from extrudate shape to pipe shape in Figure 16.

\section{IV.RESULTS}

Using Eq. (55), we plot Figure 11, from which we learn that a Newtonian extrudate will never knuckle. Extrudate knuckling thus comes from the nonNewtonian contribution. Using Eq. (56), we plot Figure 12, from which we learn that the fluid elasticity, identified by $\sigma$, causes extrudate knuckling. This is why, to avoid knuckles, engineers must sometimes reformulate the plastics.

From Figure 11 and Figure 12, we also learn that extrudate knuckling never happens in the upper quadrants $(\pi / 2>\tilde{\theta}>3 \pi / 2)$ of the pipe. Thus, when extrudate knuckling does happen, it always happens in the lower quadrants $(\pi / 2 \leq \tilde{\theta} \leq 3 \pi / 2)$. These findings agree with industrial experience.

From Figure 13, we learn that the critical value of $(1-\sigma) S$ above which extrudate knuckling happens, $\mathbb{K}$, descends with $\xi_{i}$ and increases with $\xi_{0}$. For any pair $\left(\xi_{i}, \xi_{o}\right)$, the value of $(1-\sigma) S$ must subceed the value of $\mathbb{K}$ interpolated from Figure 13:

$(1-\sigma) S<\mathbb{K}$

which is working inequality for suppressing extrudate knuckling. Eq. (69), along with its companion Figure 13, illustrates the extrudate knuckling criterion (Eqs. (59), (60) and (61) [or Eq. (62)]), which is the main result of this paper.

To deepen the reader's understanding of how Figure 13 was constructed, we provide Figure 14, and we recall: 
$\frac{\partial\left\langle\breve{v}_{z}\right\rangle_{\theta}}{\partial \theta}=\frac{\partial\left\langle\breve{v}_{z}\right\rangle_{\theta, 0}}{\partial \theta}+(1-\sigma) S \frac{\partial\left\langle\breve{v}_{z}\right\rangle_{\theta, 1}}{\partial \theta}$

where $\partial\left\langle\breve{v}_{z}\right\rangle_{\theta} / \partial \theta$ is the extrudate shape gradient (ESG), $\partial\left\langle\breve{v}_{z}\right\rangle_{\theta, 0} / \partial \theta$ is the Newtonian contribution to the ESG, and $\partial\left\langle\breve{v}_{z}\right\rangle_{\theta, 1} / \partial \theta$ is part of the nonNewtonian contribution. Specifically, Figure 14 shows how one point, the red point on the ordinate $\left(\xi_{i}, \mathbb{K}\right)=(0.01,0.018)$, in Figure 13 was obtained. When $\partial\left\langle\breve{v}_{z}\right\rangle_{\theta, 0} / \partial \theta$ intersects $\partial\left\langle\breve{v}_{z}\right\rangle_{\theta, 1} / \partial \theta$ at $\theta<\pi,(1-\sigma) S>\mathbb{K}$ (see, for example, the isopleth of $(1-\sigma) S=0.01)$. When $\partial\left\langle\breve{v}_{z}\right\rangle_{\theta, 0} / \partial \theta$ and $\partial\left\langle\breve{v}_{z}\right\rangle_{\theta, 1} / \partial \theta$ do not intersect, $(1-\sigma) S<\mathbb{K}$ (see, for example, the isopleth of $(1-\sigma) S=0.001$ ). When $\partial\left\langle\breve{v}_{z}\right\rangle_{\theta, 0} / \partial \theta$ intersects $\partial\left\langle\breve{v}_{z}\right\rangle_{\theta, 1} / \partial \theta$ at precisely $\theta=\pi,(1-\sigma) S=\mathbb{K}$ (see the $(1-\sigma) S=0.00263=\mathbb{K}$ isopleth).

Figure 15 shows that extrudate knuckling, predicted by Eq. (59), happens in concert with a local maximum in pipe thickness at the bottom of the pipe. From Figure 15, we also learn that increasing $(1-\sigma) S$ worsens the extrudate knuckling. Figure 16 compares the initial condition normally assumed for numerical simulation of sag (red curve) [6], with the initial condition that this paper offers (blue curve): the extrudate shape entering the cooling chamber (predicted by Eq. (67) [with Eq. (47)]). Specifically, from Figure 16, we see why the initial condition that is normally assumed for numerical simulation of sag over-predicts the pipe bottom thickness. 


\section{CONCLUSION}

We employ our analytical solution for the pressure-driven flow of a nonNewtonian fluid through an eccentric annulus to analyze the shape of extrudate emerging from a pipe die. We employ the Oldroyd 8-constant model for its rich diversity of special cases. We find melt viscoelasticity to be a necessary condition for extrudate knuckling. Further, we arrive at a new criterion for extrudate knuckling (Eqs. (59), (60) and (61) [or Eq. (62)]). This criterion yields a working inequality, Eq. (69), for suppressing extrudate knuckling. Figure 13 illustrates this new working inequality, and plastics engineers can use it to predict extrudate knuckling.

This paper offers significant improvement over the initial condition normally used for numerical simulation of sag in plastic pipe extrusion. The new initial condition not only includes extrudate knuckling, but also offers a promising solution to the over-prediction of the pipe bottom thickness (see Figure 16; and Fig. 6 of [6]).

\section{VI.WORKED EXAMPLE}

An engineer observes the pipe knuckling (shown in Figure 10) in highdensity polyethylene pipe extrusion. For sag compensation, her mandrel has been shifted downward by $\delta=0.0118 \mathrm{~m}$, with mandrel and die radii of $R_{i}=0.351 \mathrm{~m}$ and $R_{o}=0.440 \mathrm{~m}$. For her analysis, the engineer choses the corotational Maxwell model (see Subsection I.b; also [45,46]), with $\eta_{0}=9.6 \mathrm{MPa} \cdot \mathrm{s}$ and $\lambda_{1}=2.38 \mathrm{~s}$ for her molten high-density polyethylene. Does extrudate knuckling cause her pipe knuckling? 
She first calculates the corresponding inner and outer contours by substituting $R_{i}, R_{o}$ and $\delta$ into Eqs. (7), (36) and (37) to get:

$$
a=5.91 \mathrm{~m}
$$

$\xi_{i}=0.0592$

and:

$\xi_{o}=0.0740$

She measures the pressure drop per unit length across her annular die of:

$P=8.46 \times 10^{5} \mathrm{~Pa} / \mathrm{m}$

Using Eq. (73) and the given material properties to calculate the non-

Newtonian coefficient, $(1-\sigma) S$, gives:

$$
(1-\sigma) S=\frac{(2.38 \mathrm{~s})^{2}(5.91 \mathrm{~m})^{2}\left(8.46 \times 10^{5} \mathrm{~Pa} / \mathrm{m}\right)^{2}}{16\left(9.6 \times 10^{6} \mathrm{~Pa} \cdot \mathrm{s}\right)^{2}}=0.0960
$$

Next, she uses Eqs. (71) and (72) to interpolate the critical value of $(1-\sigma) S$ from Figure 13 to get:

$\mathbb{K}=0.00557$

Since this is smaller than 0.0246 , she concludes that her pipe knuckling is indeed caused by the knuckling of her extrudate.

\section{ACKNOWLEDGMENT}

The financial support of the Royal Golden Jubilee Program of the Thailand Research Fund for (Contract No. PHD/0116/2554) is also greatly appreciated. A.J. Giacomin is indebted to the Faculty of Applied Science and Engineering of Queen's University at Kingston, for its support through a Research Initiation 
Grant (RIG). This research was undertaken, in part, thanks to support from the Canada Research Chairs program of the Government of Canada for the Natural Sciences and Engineering Research Council of Canada (NSERC) Tier 1 Canada Research Chair in Rheology.

\section{VIII.APPENDIX}

In this appendix, we derive the relationship between the cylindrical angular, $\tilde{\theta}$ and eccentric cylindrical angular coordinates $\theta$. We begin by relating the Cartesian coordinate $x$ in Figure 8 (to which the eccentric cylindrical coordinates are referred) with the Cartesian coordinate $\tilde{x}$ in Figure 7 (to which the cylindrical coordinates are referred):

$x=\tilde{x}+x_{\mathrm{c}}$

where the circle center is given by $x_{c}=a /\left(1-\xi^{2}\right)$ (from Eq. (6) of [12]).

Substituting this into Eq. (76) gives:

$x=\tilde{x}+\frac{a}{1-\xi_{o}^{2}}$

where (see Eq. (A.6-1) in [23]):

$\tilde{x} \equiv r \cos \tilde{\theta}$

Substituting Eqs. (1) and (78) into Eq. (77), and solving for $\theta$ gives:

$\theta=\arccos \left(\frac{\left(1+\xi^{2}\right)\left(\frac{R}{a} \cos \tilde{\theta}+\frac{1}{1-\xi^{2}}\right)-1}{2 \xi\left(\frac{R}{a} \cos \tilde{\theta}+\frac{1}{1-\xi^{2}}\right)-\xi}\right)$

Since the origin of the cylindrical coordinates is at the outer circle center, Eq.

(79) becomes: 
$\theta=\arccos \left(\frac{\left(1+\xi_{o}^{2}\right)\left(\frac{R_{o}}{a} \cos \tilde{\theta}+\frac{1}{1-\xi_{o}^{2}}\right)-1}{2 \xi_{o}\left(\frac{R_{o}}{a} \cos \tilde{\theta}+\frac{1}{1-\xi_{o}^{2}}\right)-\xi_{0}}\right)$

or for $\tilde{\theta}$ :

$\tilde{\theta}=\arccos \left(\frac{a}{R_{o}} \frac{1-\xi_{o} \cos \theta}{1-2 \xi_{o} \cos \theta+\xi_{o}^{2}}-\frac{a}{R_{o}} \frac{1}{1-\xi_{o}^{2}}\right)$

Eqs. (80) [Eq. (81)] can be used to convert from $\theta$ to $\tilde{\theta}$ [vice versa]. 
Table 1: Dimensional Variables

\begin{tabular}{|l|l|l|}
\hline \multicolumn{1}{|c|}{ Name } & Unit & \multicolumn{1}{c|}{ Symbol } \\
\hline Any coordinates, $i$-th & $L$ & $x_{i}$ \\
\hline Any tensor, $i$-th component & & $b_{i k}$ \\
\hline Area, slice $\tilde{\theta}$ of each extrudate & $L^{2}$ & $A_{\mathrm{e} \tilde{\theta}}$ \\
\hline $\begin{array}{l}\text { Area, slice } \tilde{\theta} \text { of each postdie } \\
\text { extrudate that entering cooling } \\
\text { chamber }\end{array}$ & $L^{2}$ & $A_{\mathrm{p} \tilde{\theta}}$ \\
\hline Area, $\xi \theta$ - plane & $L^{2}$ & $A_{\xi \theta}$ \\
\hline Axial velocity & $L / t$ & $v_{z}$ \\
\hline Cartesian coordinates & $L$ & $x, y, z_{\mathrm{C}}$ \\
\hline Confocal length & $L$ & $a$ \\
\hline Corotational derivative & $t^{-1}$ & $\mathscr{Q} / \mathscr{Q}) t$ \\
\hline Density & $M / L^{3}$ & $\rho$ \\
\hline $\begin{array}{l}\text { Eccentric cyl indrical coordinate, } \\
\text { axial direction }\end{array}$ & $L$ & $z$ \\
\hline Eccentricity & $L$ & $\delta$ \\
\hline Extra stress tensor & $M / L t^{2}$ & $\tau_{i k}$ \\
\hline First normal stress coefficient & $M / L$ & $\Psi_{1} \equiv\left(\tau_{11}-\tau_{22}\right) / \dot{\gamma}^{2}$ \\
\hline $\begin{array}{l}\text { First normal stress coefficient, zero } \\
\text { shear rate }\end{array}$ & $M / L$ & $\Psi_{10} \equiv 2 \eta_{0}\left(\lambda_{1}-\lambda_{2}\right)$ \\
\hline Infinite shear rate viscosity & $M / L t$ & $\eta_{\infty}$ \\
\hline $\begin{array}{l}\text { Magnitude of the rate of } \\
\text { deformation tensor }\end{array}$ & $t^{-1}$ & $\dot{\gamma}$ \\
\hline $\begin{array}{l}\text { Magnitude of the rate of } \\
\text { deformation tensor, inflection point }\end{array}$ & $t^{-1}$ & $\dot{\gamma}_{c}$ \\
\hline Oldroyd coefficient & $t$ & $\mu_{0}$ \\
\hline Oldroyd coefficient & $t$ & $\mu_{1}$ \\
\hline Oldroyd coefficient & $t$ & $\mu_{2}$ \\
\hline Oldroyd coefficient & $t$ & $v_{1}$ \\
\hline Oldroyd coefficient & $M / L t^{2}$ & $v_{2}$ \\
\hline Oldroyd coefficient, relaxation time & $t$ & $\lambda_{1}$ \\
\hline Oldroyd coefficient, retardation time & $t$ & $\lambda_{2}$ \\
\hline Oldroyd shear thickening constant & $t^{2}$ & $\sigma_{2}$ \\
\hline Oldroyd shear thinning constant & $t^{2}$ & $\sigma_{1}$ \\
\hline Pressure gradient in die land & $L / d z$ \\
\hline Radius, die & & \\
\hline Radius, mandrel & & \\
\hline
\end{tabular}




\begin{tabular}{|l|l|l|}
\hline $\begin{array}{l}\text { Radius, postdie extrudate, entering } \\
\text { cooling chamber }\end{array}$ & $L$ & $R_{i \mathrm{p}}$ \\
\hline $\begin{array}{l}\text { Radius, postdie extrudate, entering } \\
\text { cooling chamber }\end{array}$ & $L$ & $R_{o \mathrm{p}}$ \\
\hline Rate of deformation tensor & $t^{-1}$ & $\dot{\gamma}_{i k}$ \\
\hline $\begin{array}{l}\text { Scale factor, eccentric angular } \\
\text { cylindrical coordinate }\end{array}$ & $L$ & $h_{\theta}$ \\
\hline $\begin{array}{l}\text { Scale factor, eccentric radial } \\
\text { cylindrical coordinate }\end{array}$ & $L$ & $h_{\xi}$ \\
\hline Second normal stress coefficient & $M / L$ & $\Psi_{2} \equiv\left(\tau_{22}-\tau_{33}\right) / \dot{\gamma}^{2}$ \\
\hline $\begin{array}{l}\text { Second normal stress coefficient, } \\
\text { zero shear rate }\end{array}$ & $M / L$ & $\Psi_{20} \equiv-\eta_{0}\left(\lambda_{1}-\lambda_{2}-\mu_{1}+\mu_{2}\right)$ \\
\hline Steady shear viscosity function & $M / L t$ & $\eta$ \\
\hline $\begin{array}{l}\text { Steady shear viscosity, viscosity } \\
\text { curve inflection }\end{array}$ & $M / L t$ & $\eta_{\mathrm{i}}$ \\
\hline $\begin{array}{l}\text { Thickness, postdie extrudate, } \\
\text { entering cooling chamber }\end{array}$ & $L$ & $\Delta_{\mathrm{p}}$ \\
\hline Thickness, extrudate & $L$ & $\Delta$ \\
\hline Total stress tensor, $i j$-th component & $M / L t^{2}$ & $\pi_{i j}$ \\
\hline Velocity, characteristic & $L / t$ & $W \equiv a^{2} P / 4 \eta_{0}$ \\
\hline Velocity, drawdown & $L / t$ & $V$ \\
\hline Vorticity tensor, $i j$-th component & $t^{-1}$ & $\omega_{i j}$ \\
\hline Zero shear viscosity & $M / L t$ & $\eta_{0}$ \\
\hline
\end{tabular}

Legend: $M \equiv$ mass; $L \equiv$ length; $t \equiv$ time; $T \equiv$ temperature 
Table 2: Dimensionless Variables and Groups

\begin{tabular}{|c|c|}
\hline Name & Symbol \\
\hline Aspect ratio & $\kappa \equiv R_{i} / R_{o}$ \\
\hline Average velocity at angular position $\theta$ & $\left\langle\breve{v}_{z}\right\rangle_{\theta}$ \\
\hline Average velocity at angular position $\tilde{\theta}$ & $\left\langle\breve{v}_{z}\right\rangle_{\tilde{\theta}}$ \\
\hline $\begin{array}{l}\text { Average velocity at angular position } \theta \text {, Newtonian } \\
\text { contribution }\end{array}$ & $\left\langle\breve{v}_{z}\right\rangle_{\theta, 0}$ \\
\hline $\begin{array}{l}\text { Average velocity at angular position } \theta, \text { non- } \\
\text { Newtonian variable }\end{array}$ & $\left\langle\breve{v}_{z}\right\rangle_{\theta, 1}$ \\
\hline Axial velocity & $\breve{v}_{z} \equiv 4 \eta_{0} v_{z} / a^{2} P$ \\
\hline Axial velocity, Newtonian contribution & $v_{0}$ \\
\hline Axial velocity, non-Newtonian variable & $v_{1}$ \\
\hline $\begin{array}{l}\text { Axial velocity, non-Newtonian variable, particular } \\
\text { solution, } m \text {-th group, } n \text {-th harmonic }\end{array}$ & $v_{1 p, n}^{(m)}$ \\
\hline $\begin{array}{l}\text { Constant, } m \text {-th group, } \\
n \text {-th harmonic, } \ell \text {-th element }\end{array}$ & $C_{n, \ell}^{(m)}$ \\
\hline Cylindrical coordinate, angular & $\tilde{\theta}$ \\
\hline Drawdown ratio & $\mathrm{DDR} \equiv\left\langle\breve{v}_{z}\right\rangle_{\tilde{\theta}} / \breve{V}$ \\
\hline Eccentric angular cylindrical coordinate & $\theta$ \\
\hline $\begin{array}{l}\text { Eccentric angular cylindrical coordinate, extrudate } \\
\text { knuckling. }\end{array}$ & $\theta_{\mathrm{K}}$ \\
\hline Eccentric radial cylindrical coordinate & $\xi$ \\
\hline $\begin{array}{l}\text { Eccentric radial cylindrical coordinate, inner } \\
\text { contour }\end{array}$ & $\xi_{i}$ \\
\hline $\begin{array}{l}\text { Eccentric radial cylindrical coordinate, outer } \\
\text { contour }\end{array}$ & $\xi_{0}$ \\
\hline Eccentricity & $\epsilon \equiv \delta / R_{o}$ \\
\hline $\begin{array}{l}\text { Integration coefficient, Newtonian contribution, } \\
n \text {-th harmonic }\end{array}$ & $\alpha_{n}$ \\
\hline $\begin{array}{l}\text { Integration coefficient, Newtonian contribution, } \\
n \text {-th harmonic }\end{array}$ & $\beta_{n}$ \\
\hline $\begin{array}{l}\text { Integration coefficient, non-Newtonian variable, } \\
n \text {-th harmonic }\end{array}$ & $A_{n}$ \\
\hline $\begin{array}{l}\text { Integration coefficient, non-Newtonian variable, } \\
n \text {-th harmonic }\end{array}$ & $B_{n}$ \\
\hline Kronecker delta & $\delta_{i k}$ \\
\hline Non-Newtonianness & $(1-\sigma) S$ \\
\hline Non-Newtonian coefficient, critical value & $\mathbb{K}$ \\
\hline Oldroyd constant ratio & $\sigma \equiv \sigma_{2} / \sigma_{1}=\eta_{\infty} / \eta_{0}$ \\
\hline Power-law index & $n$ \\
\hline
\end{tabular}




\begin{tabular}{|l|l|}
\hline Pressure gradient squared & $S \equiv \sigma_{1} a^{2} P^{2} / 16 \eta_{0}{ }^{2}$ \\
\hline $\begin{array}{l}\text { Spatial function of axial velocity, Newtonian } \\
\text { contribution, } n \text {-th harmonic }\end{array}$ & $\phi_{n}$ \\
\hline $\begin{array}{l}\text { Spatial function of axial velocity, non-Newtonian } \\
\text { variable, } n \text {-th harmonic }\end{array}$ & $\psi_{n}$ \\
\hline $\begin{array}{l}\text { Spatial function of axial velocity, non-Newtonian } \\
\text { variable, } n \text {-th harmonic, } m \text {-th group }\end{array}$ & $\psi_{n}^{(m)}$ \\
\hline Velocity vector & $\breve{\mathbf{v}}$ \\
\hline Velocity, pipe take-off & $\breve{V} \equiv 4 \eta_{0} V / a^{2} P$ \\
\hline Weissenberg number & $\mathrm{Wi} \equiv \sqrt{\sigma_{1}} \dot{\gamma}$ \\
\hline Weissenberg number, viscosity curve inflection & $\mathrm{Wi}_{\mathrm{i}} \equiv \sqrt{\sigma_{1}} \dot{\gamma}_{\mathrm{i}}$ \\
\hline
\end{tabular}


Table 3: Solid pipe thickness profile (see APPENDIX E of [5]).

\begin{tabular}{|c|c|c|}
\hline $\begin{array}{c}\text { Angular position in } \\
\text { cylindrical coordinates, } \tilde{\theta} \\
\text { (degrees) }\end{array}$ & $\begin{array}{c}\text { Angular position in eccentric } \\
\text { cylindrical coordinates, } \theta \\
\text { (degrees) }\end{array}$ & $\begin{array}{c}\text { Thickness } \\
\text { (millimeters) }\end{array}$ \\
\hline 0.00 & 0.00 & 73.7 \\
\hline 23.0 & 19.8 & 72.8 \\
\hline 45.0 & 39.3 & 74.0 \\
\hline 90.0 & 81.5 & 84.6 \\
\hline 125 & 118 & 94.5 \\
\hline 135 & 129 & 92.9 \\
\hline 180 & 180 & 80.1 \\
\hline 225 & 231 & 91.9 \\
\hline 270 & 279 & 80.6 \\
\hline 315 & 321 & 74.5 \\
\hline
\end{tabular}




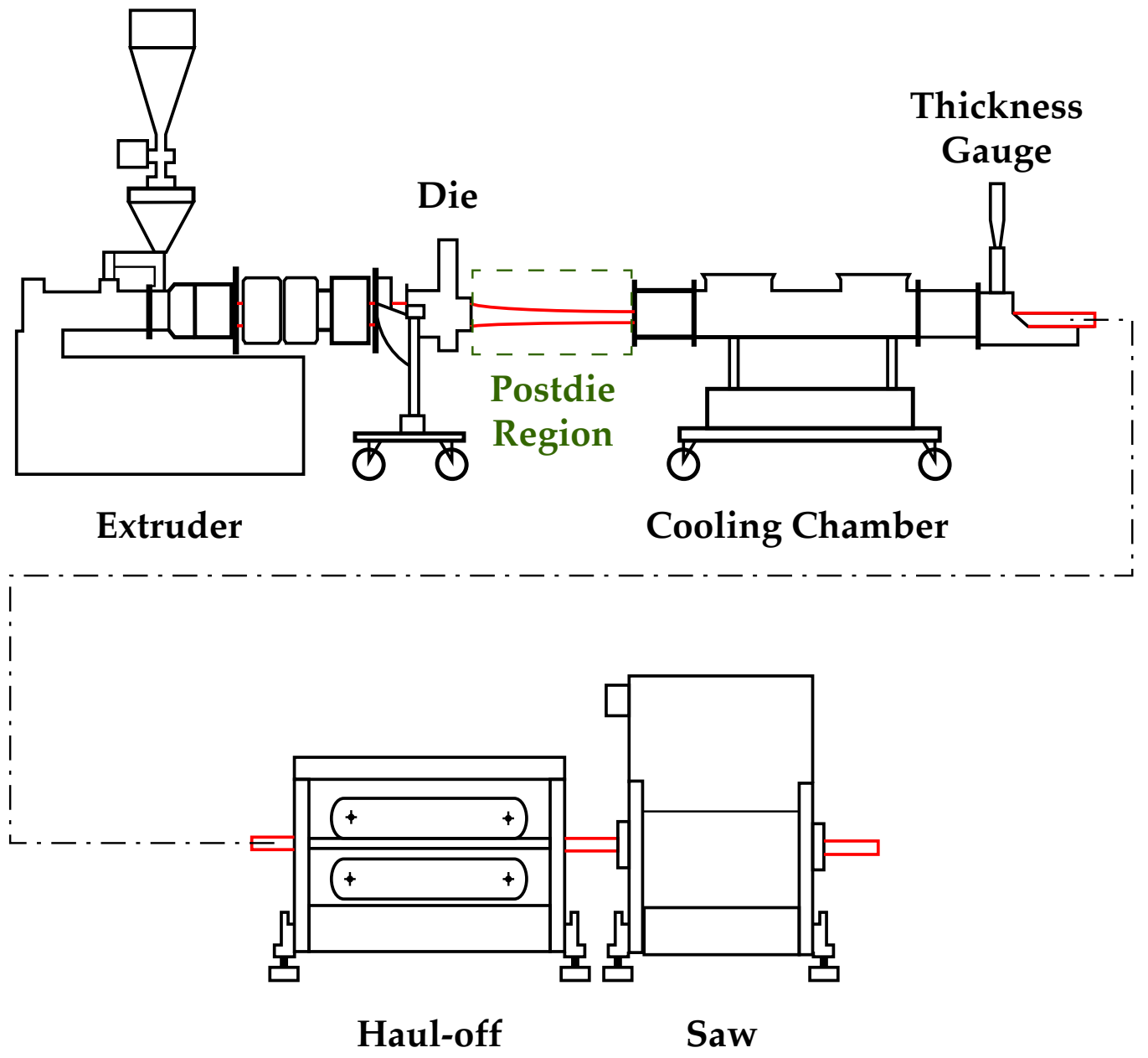

Figure 1: Pipe extrusion line. 


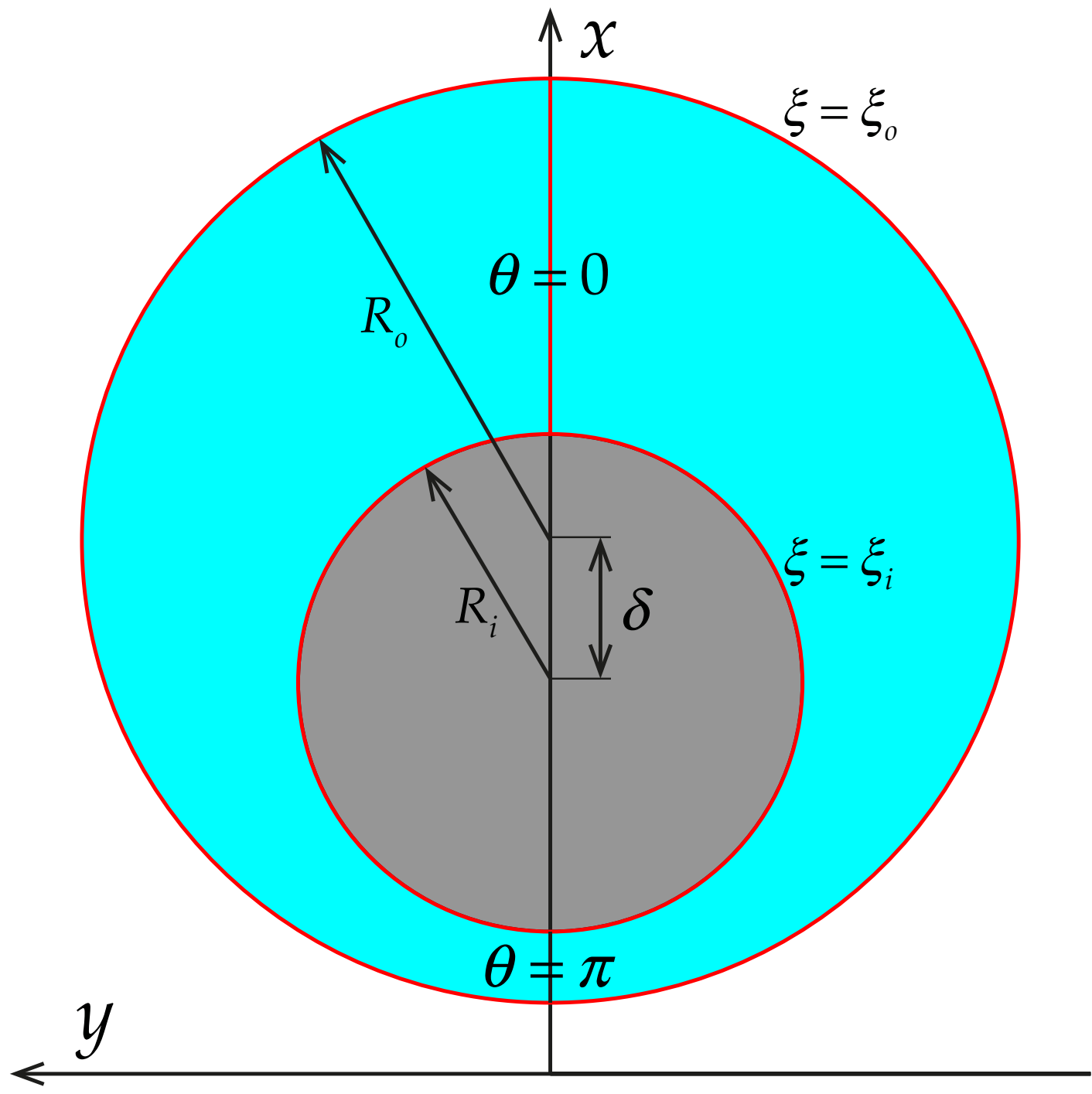

Figure 2: End view of pipe extrusion die defining the die dimensions. 


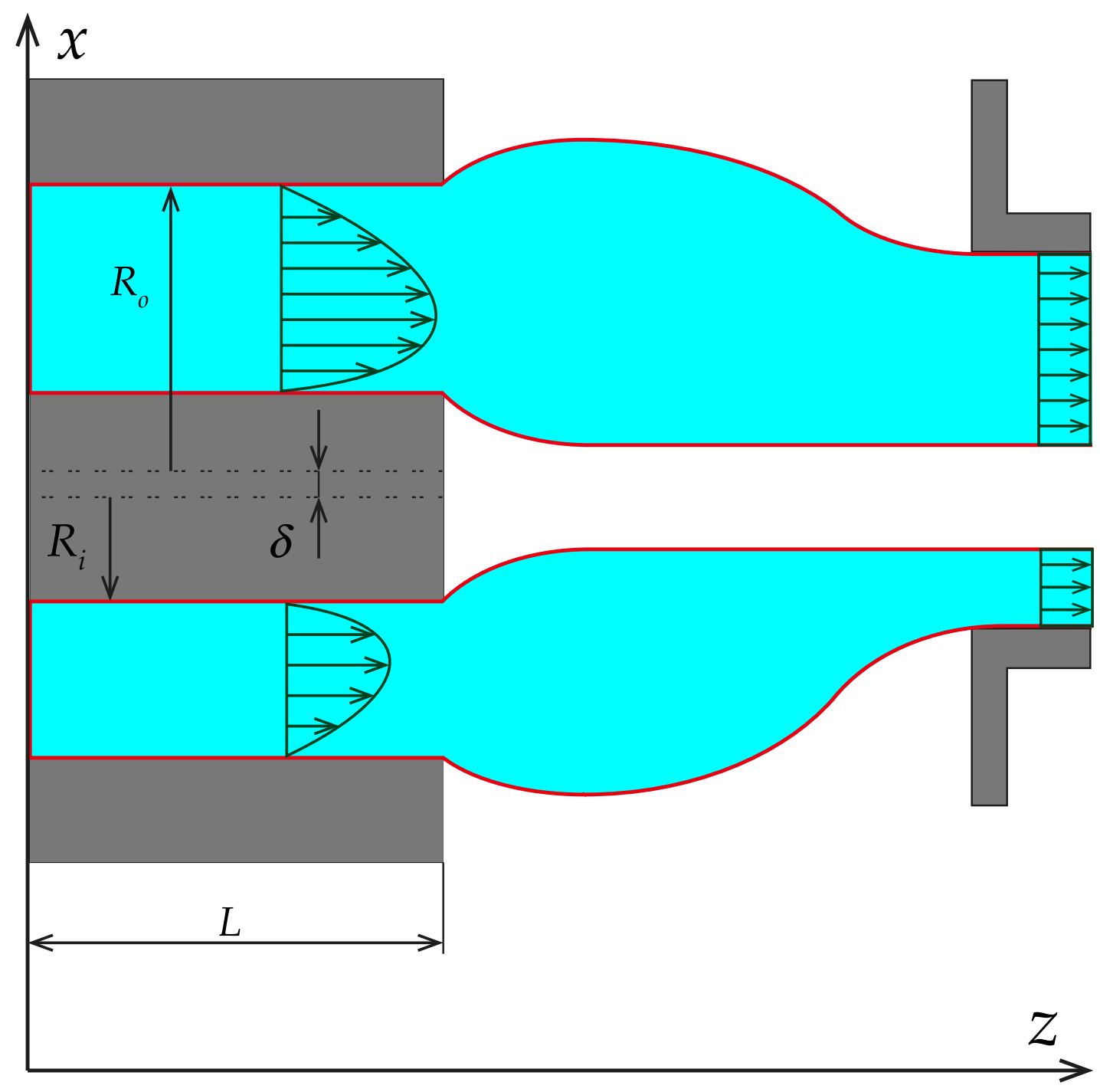

Figure 3: Side view of pipe extrusion die (left), defining the die dimensions, and the postdie region (right). The mandrel and barrel surfaces correspond to $\xi=\xi_{i}$ and $\xi=\xi_{0}$. 

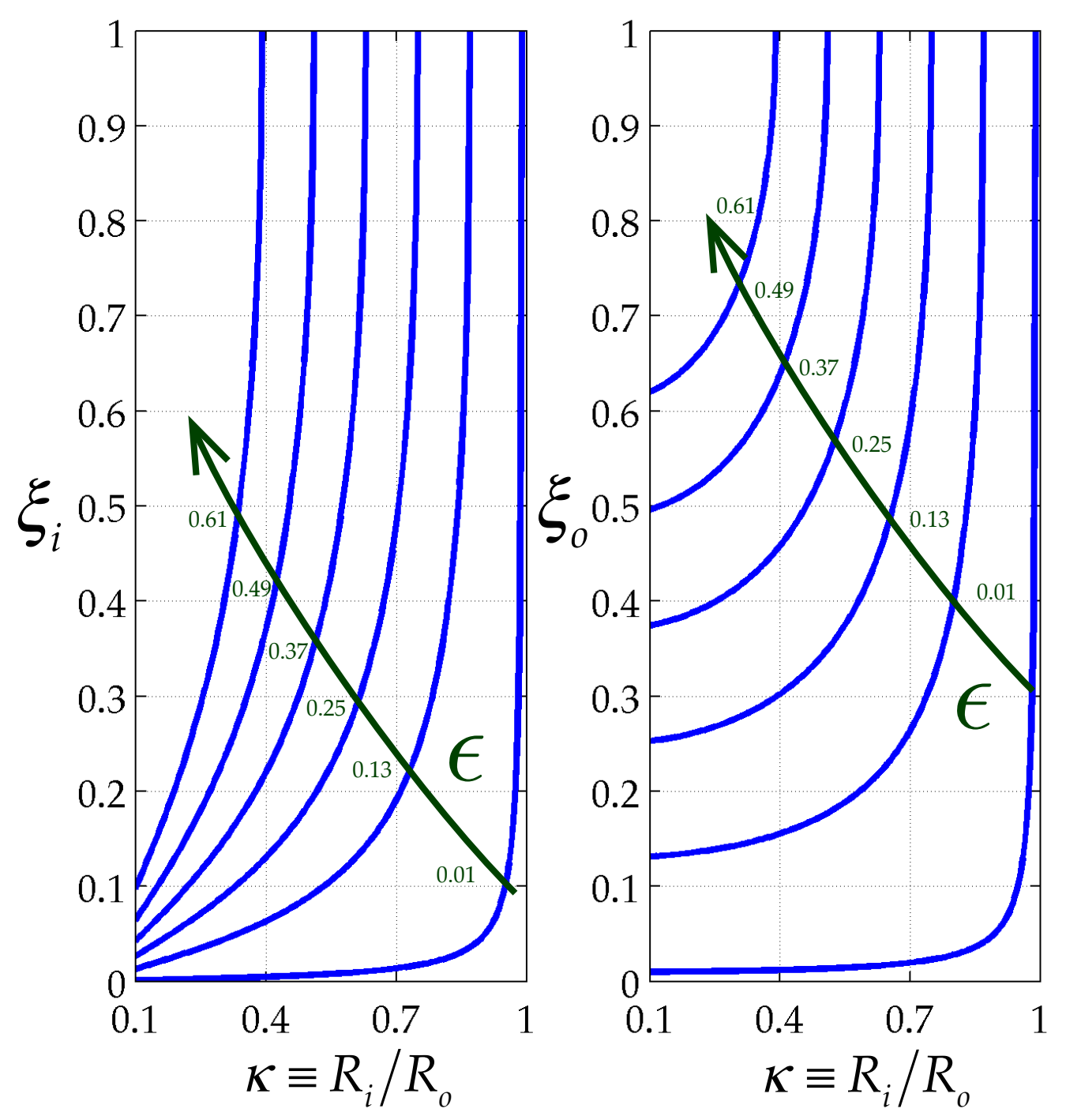

Figure 4: $\xi_{i}$ and $\xi_{o}$ versus aspect ratio, $\kappa \equiv R_{i} / R_{o}$ with curves of constant $\epsilon$. 


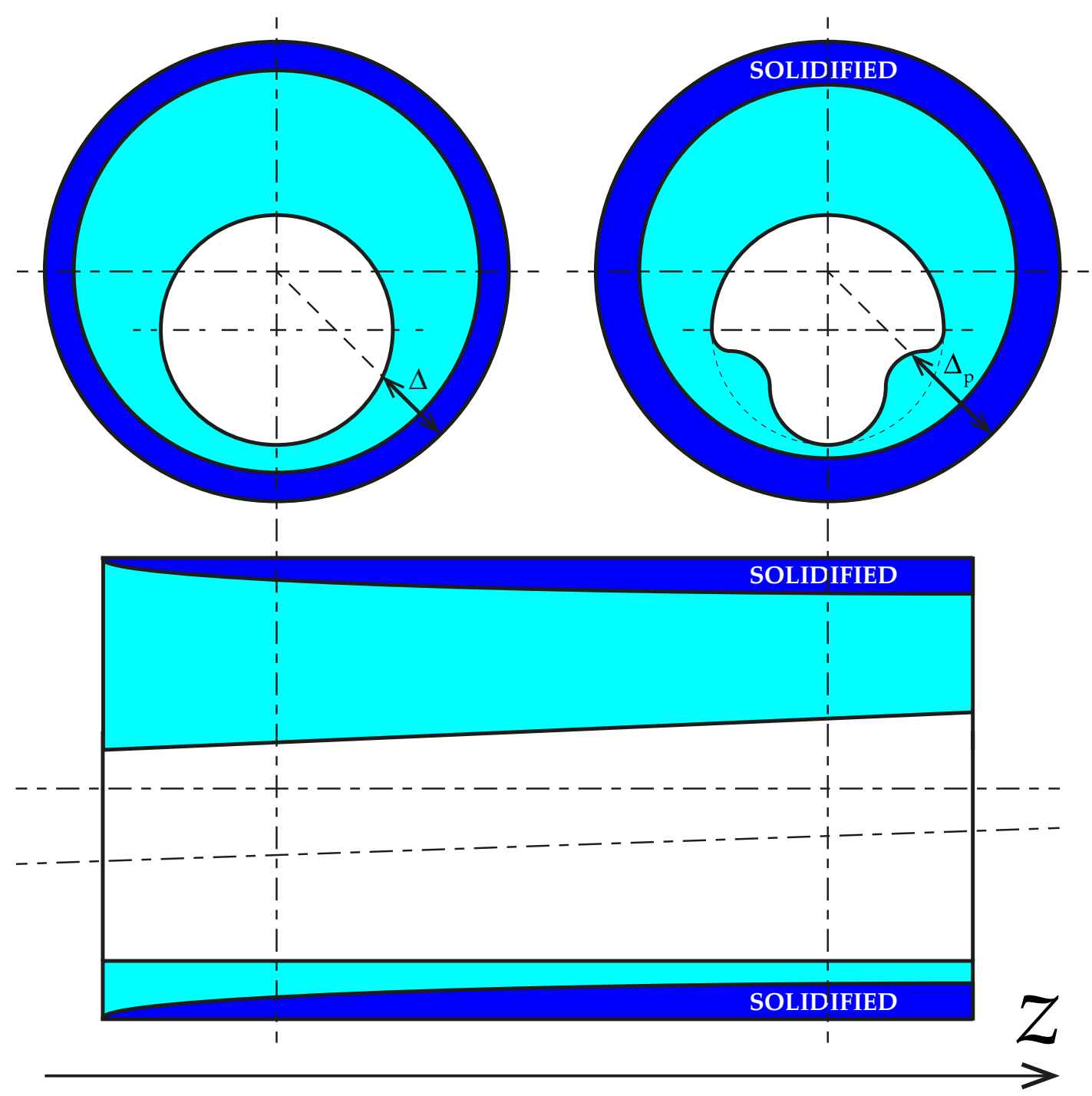

Figure 5: Knuckling from sag (slump). $R_{i}, R_{o}, \delta, \Delta$ and $\Delta_{\mathrm{p}}$ are inner and outer radii, dimensional eccentricity, the thickness of the extrudate that leaving the die, and entering the cooling chamber. 


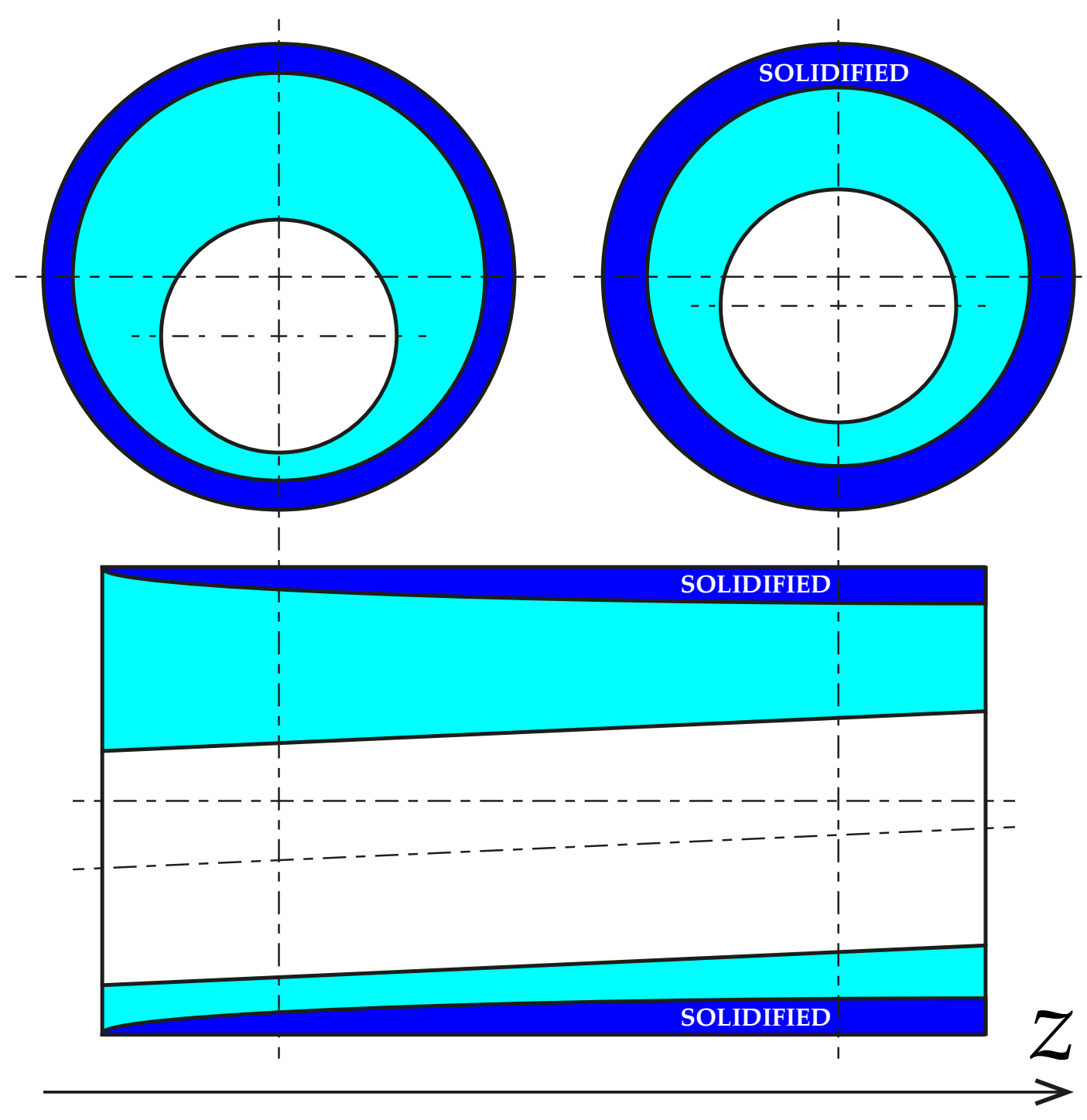

Figure 6: Sag without knuckling. 


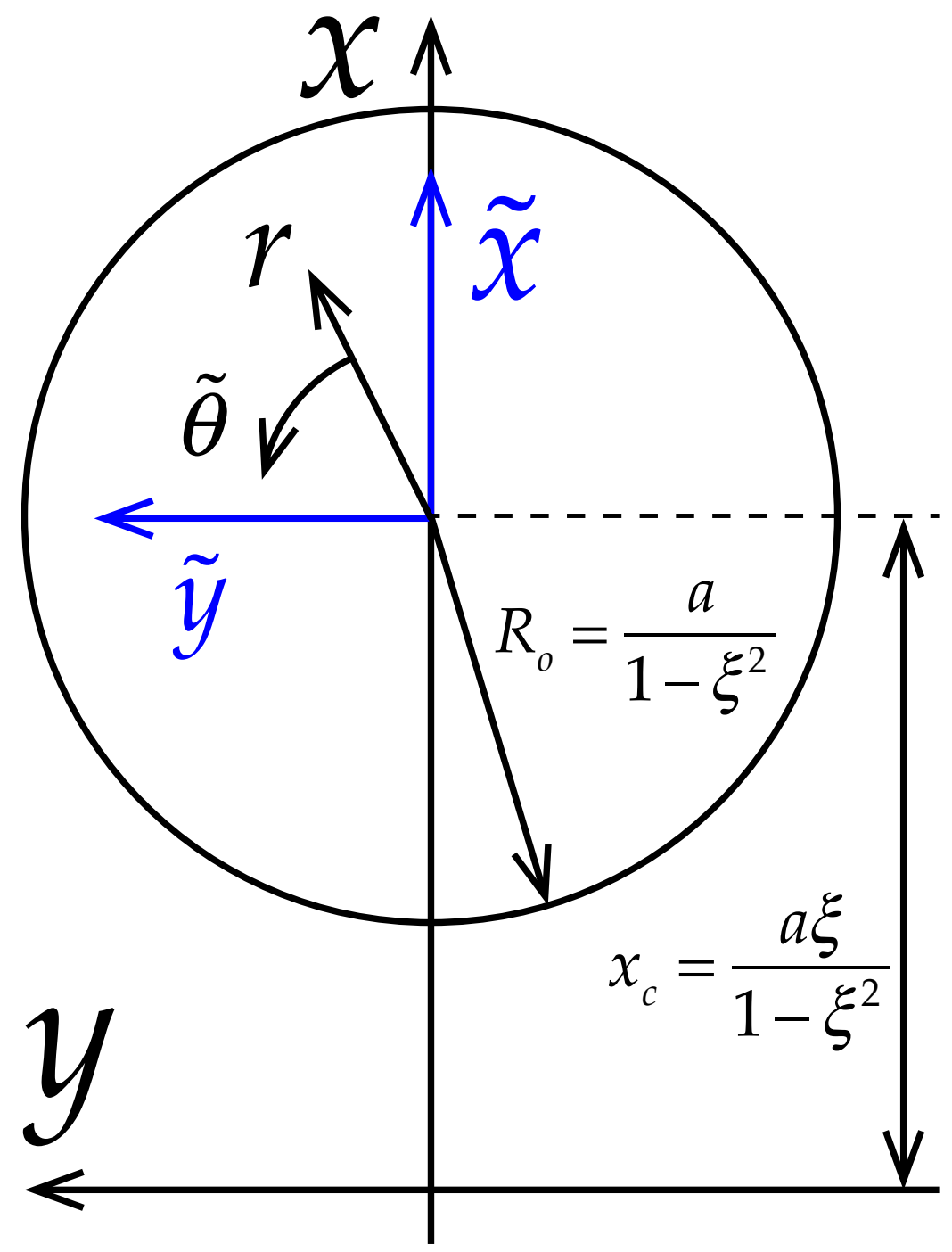

Figure 7: The shifted Cartesian coordinates, $(\tilde{x}, \tilde{y})$, versus the Cartesian coordinates in Figure 8, $(x, y)$. The origin of the cylindrical coordinates, $(r, \tilde{\theta})$, is located at $(x, y)=\left(a \xi /\left[1-\xi^{2}\right], 0\right)$. 


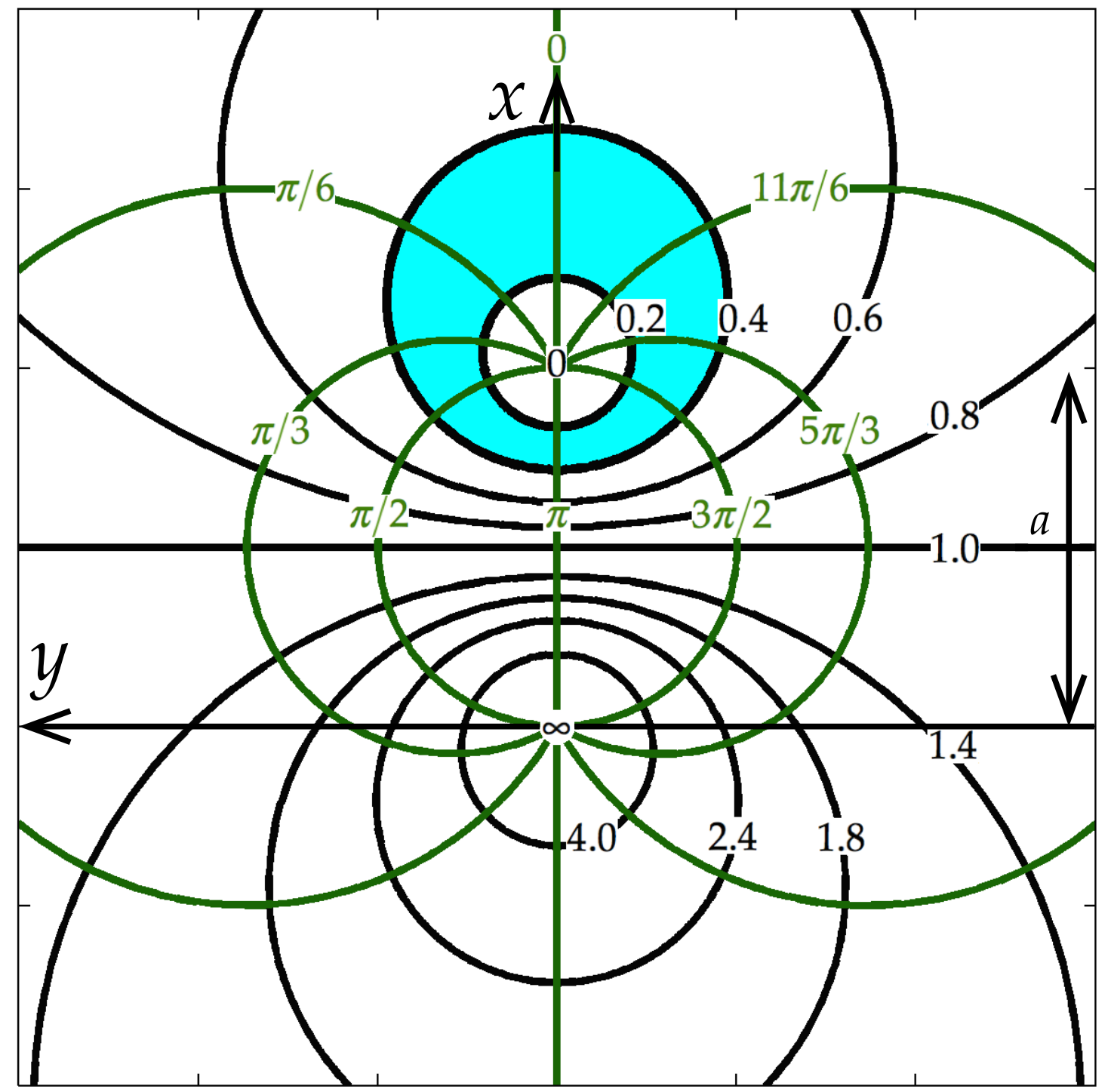

Figure 8: Eccentric cylindrical coordinates showing the circles of constant eccentric radial coordinate (black), $\xi$, and the circles of constant eccentric angular coordinate (green), $\theta$. Cyan example between $\xi=0.2$ and $\xi=0.4$. 


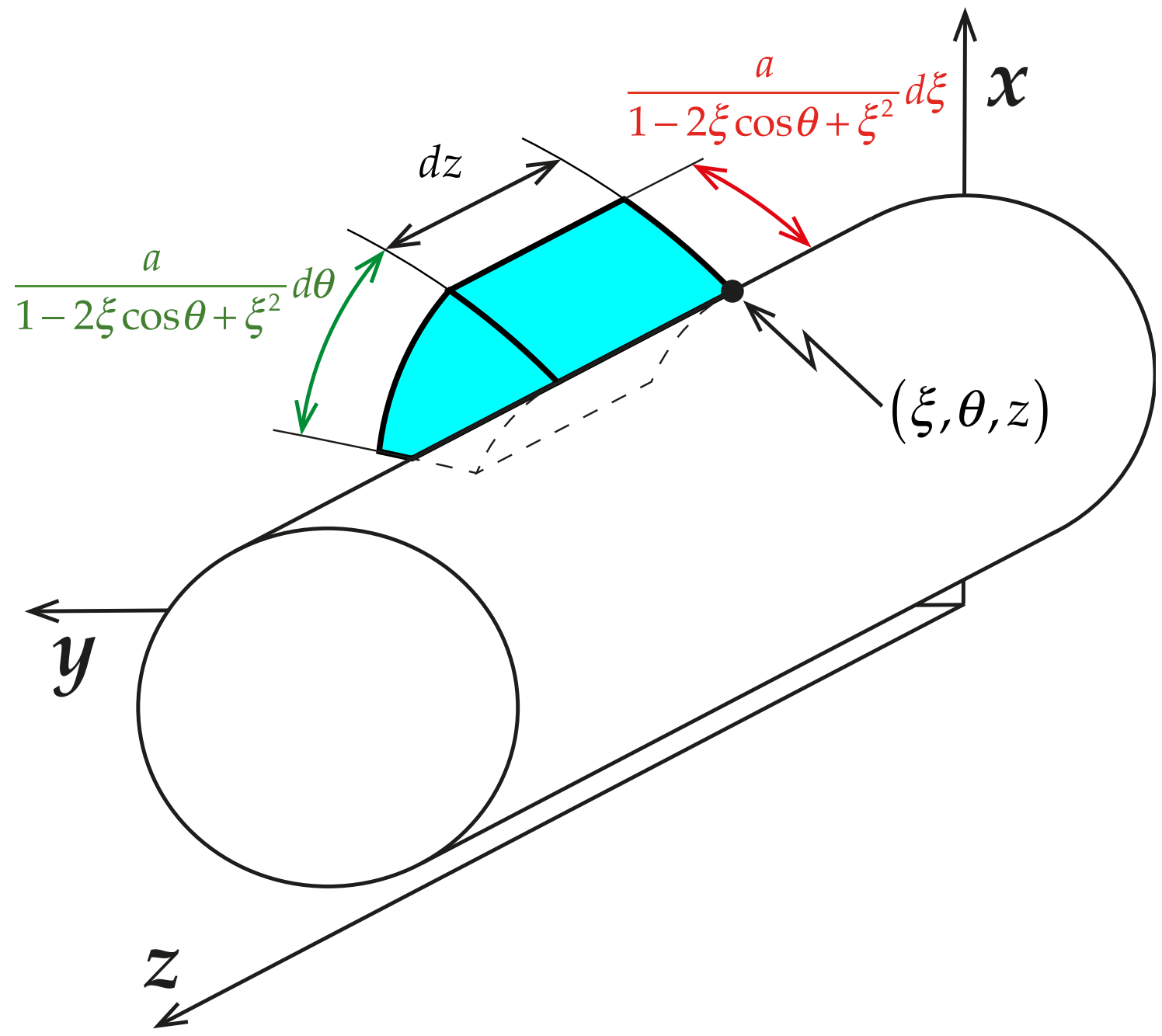

Figure 9: Differential volume element of the molten plastic in eccentric cylindrical coordinates (cyan). Edges of differential surface element through which melt flows labeled $\left[a /\left(1-2 \xi \cos \theta+\xi^{2}\right)\right] d \xi$ and $\left[a /\left(1-2 \xi \cos \theta+\xi^{2}\right)\right] d \theta$. Cylindrical shell of constant eccentric cylindrical radial coordinate $\xi$ parallels $z$-axis, and intersects $x$-axis at $x=a /\left(1-\xi^{2}\right)$. 


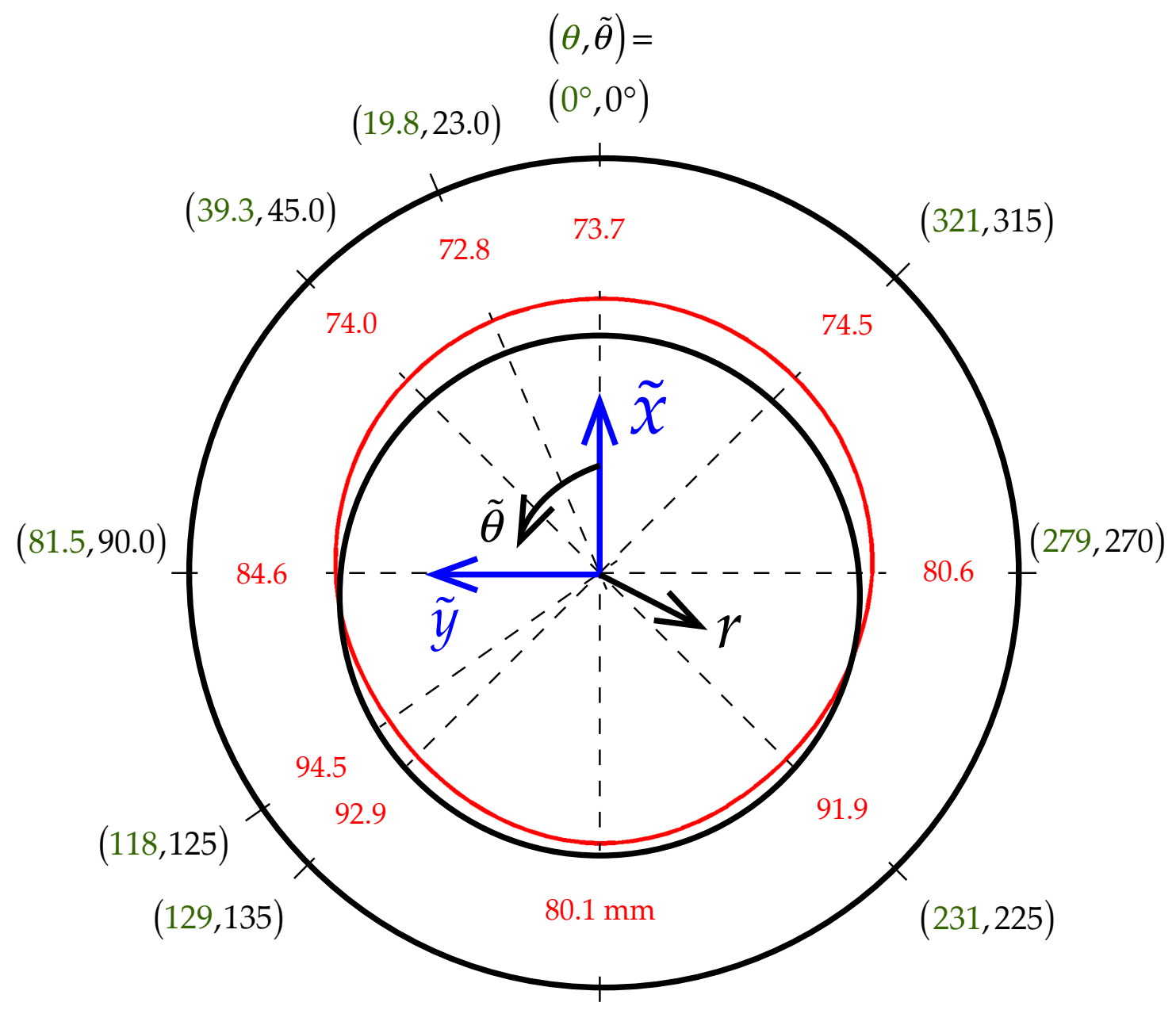

$(180,180)$

Figure 10: Measured wall thickness distribution of pipe with knuckles (red) with ten specific angular positions, for both cylindrical, $\tilde{\theta}$, and eccentric cylindrical, $\theta$, coordinates [Table 3]. Black circles represent the same eccentric die $\left(\xi_{i}, \xi_{o}\right)=(0.0592,0.0740)$ of Figure 16 . 


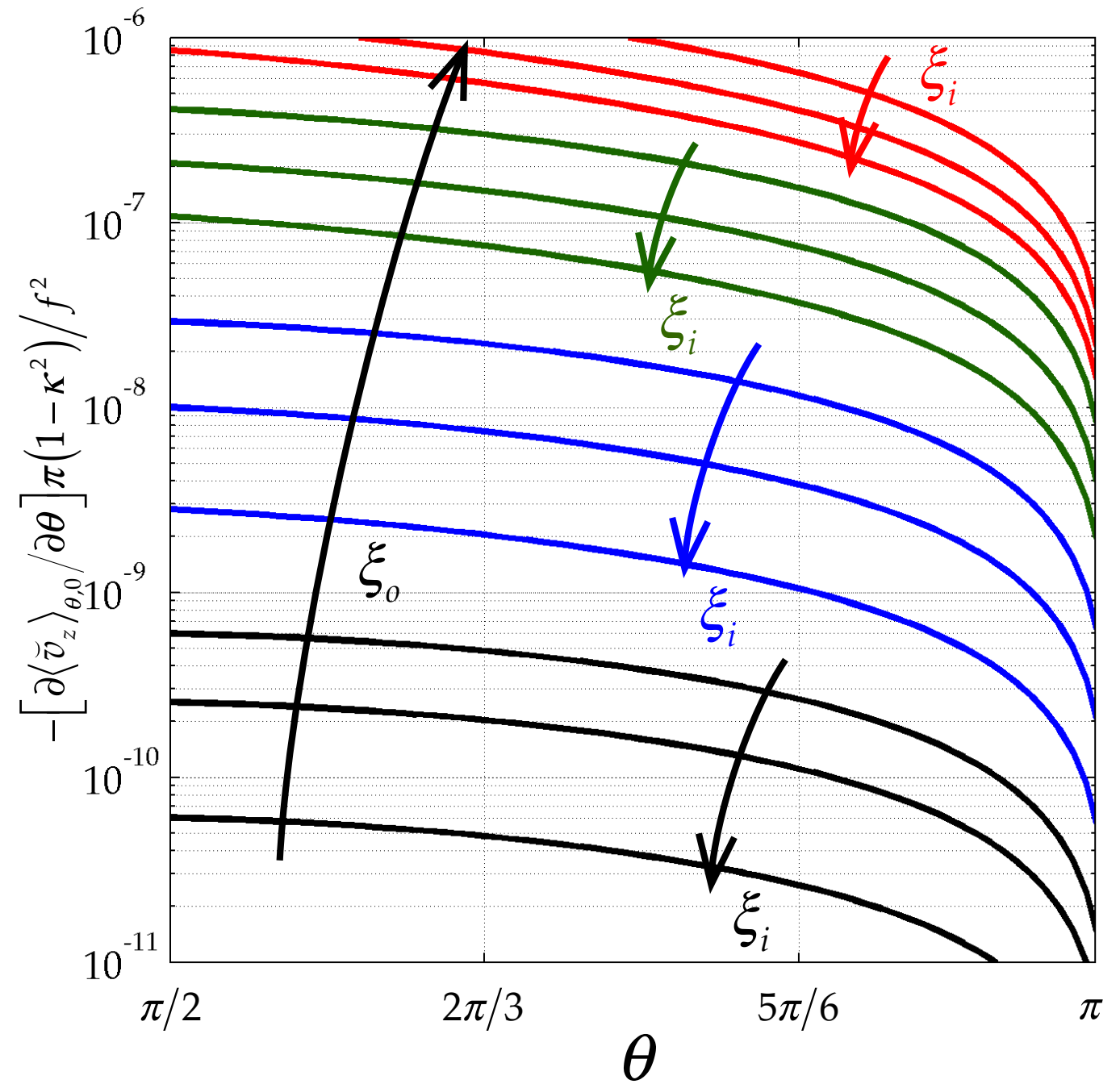

Figure 11: Newtonian contribution to the extrudate shape gradient. Four sets of increasing parametrized curves of $\xi_{i}$ from top to bottom [Eqs. (55)]. $\xi_{0}$ is increasing from bottom to top. 


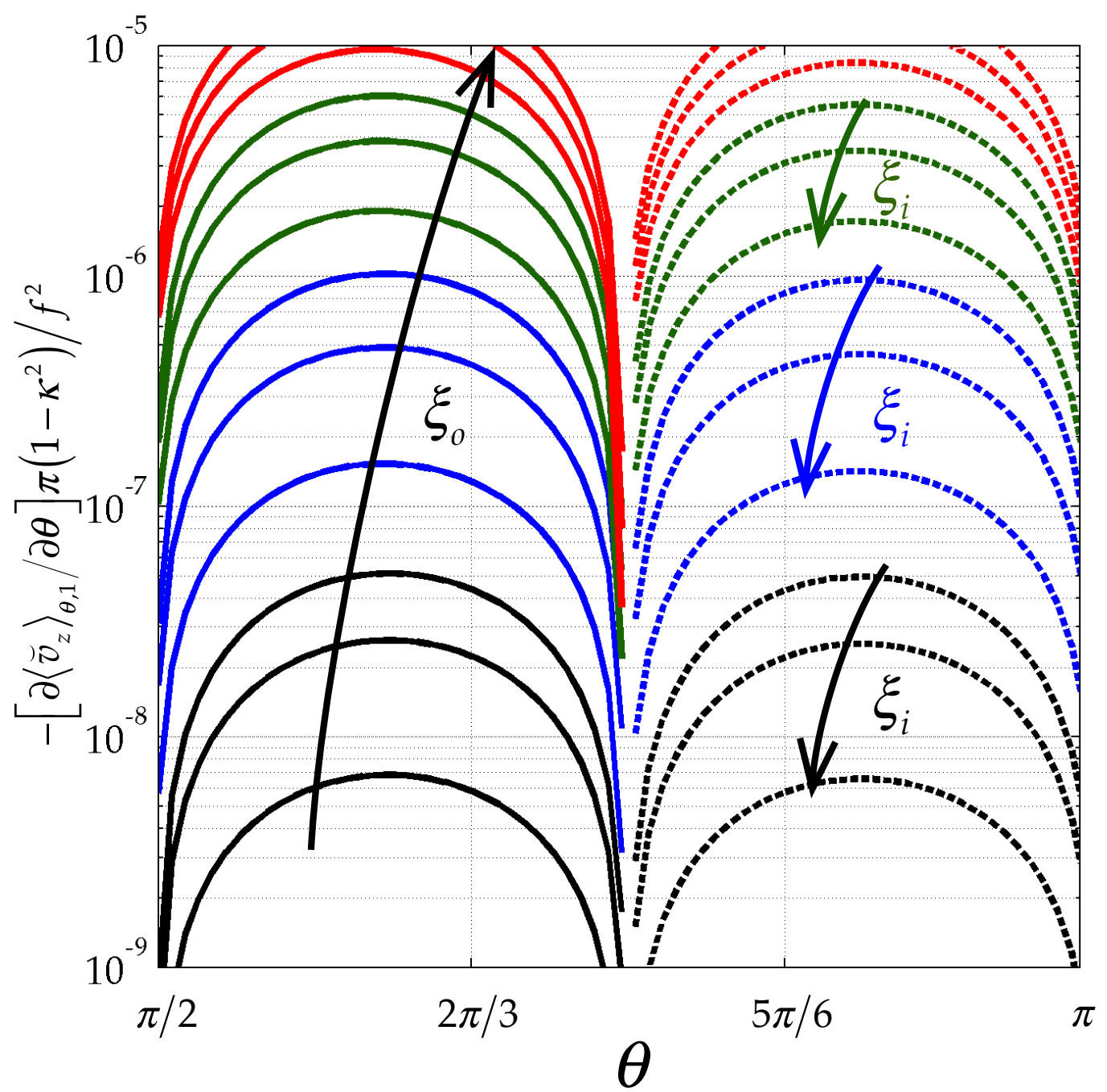

Figure 12: Non-Newtonian contribution to extrudate shape gradient. Four sets of increasing parametrized curves of $\xi_{i}$ (top to bottom). $\xi_{o}$ is increasing from bottom to top. Dashed curves are for the negative part of $-\partial\langle\breve{v}\rangle_{\theta, 1} / \partial \theta$ [Eqs. (56)]. 


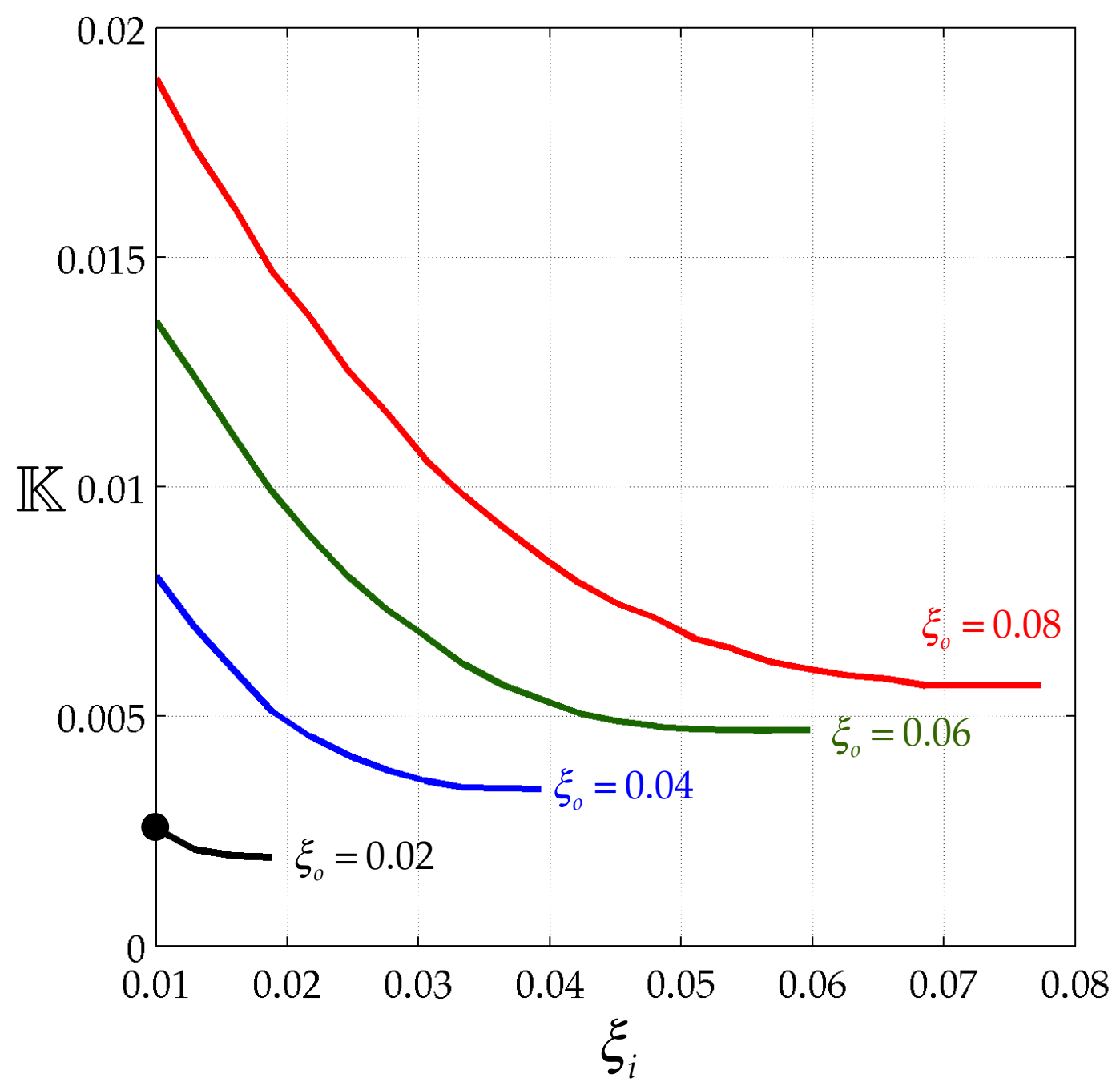

Figure 13: Knuckling suppression map of the critical values of $(1-\sigma) S, \mathbb{K}$, versus inner contour, $\xi_{i}$, parametrized with $\xi_{o}$ [Eq. (59)]. Black dot illustrates the critical value for Figure 14. 


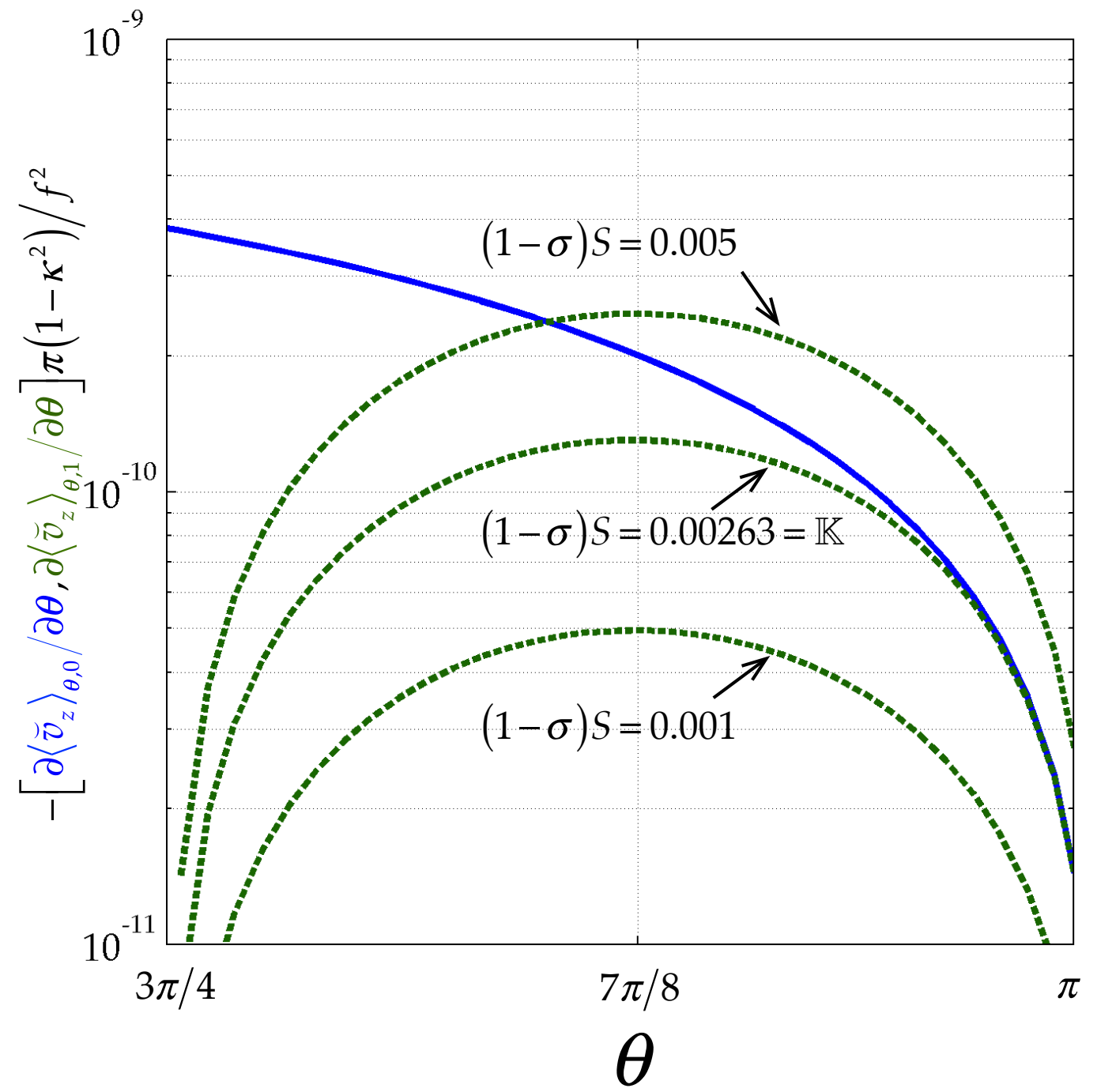

Figure 14: Suppressing extrudate knuckles. Blue curves are the Newtonian (negative) contribution to extrudate shape gradient [Eq. (55)], and the green ones, non-Newtonian [Eq. (56)], using $\left(\xi_{i}, \xi_{o}\right)=(0.01,0.02)$. Critical value is $\mathbb{K}=0.00263$. Suppressing by decreasing $(1-\sigma) S$. Dashed curves are for the negative part of $-\partial\langle\breve{v}\rangle_{\theta, 1} / \partial \theta$. 


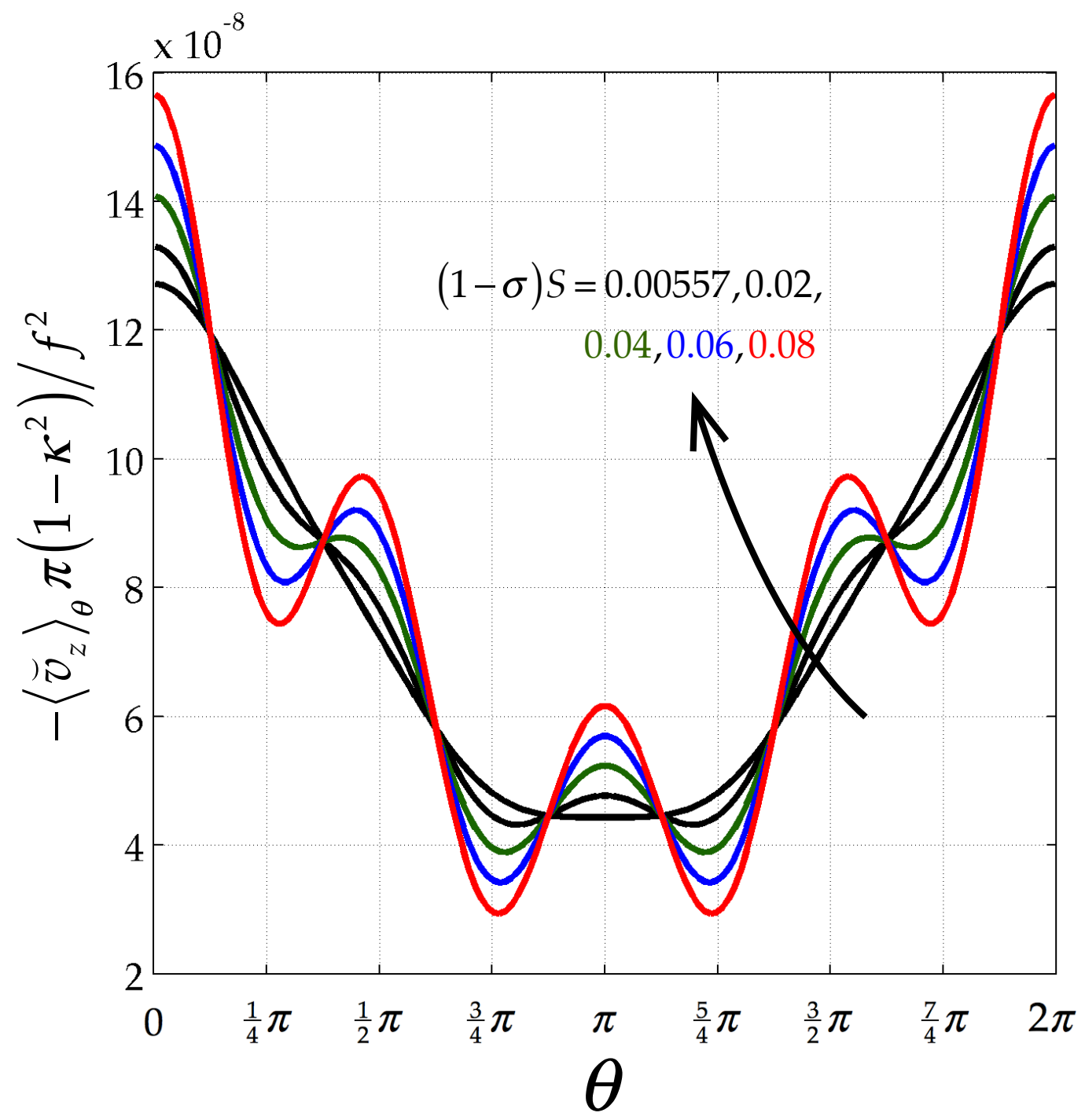

Figure 15: Average velocity of each slice $\theta$ versus eccentric angular position, $\theta$, with curves of constant $(1-\sigma) S=0.00557,0.02,0.04,0.06,0.08$ [Eq. (59)]. The smoothest curve, $(1-\sigma) S=\mathbb{K}=0.00557$, represents the critical extrudate knuckling. 


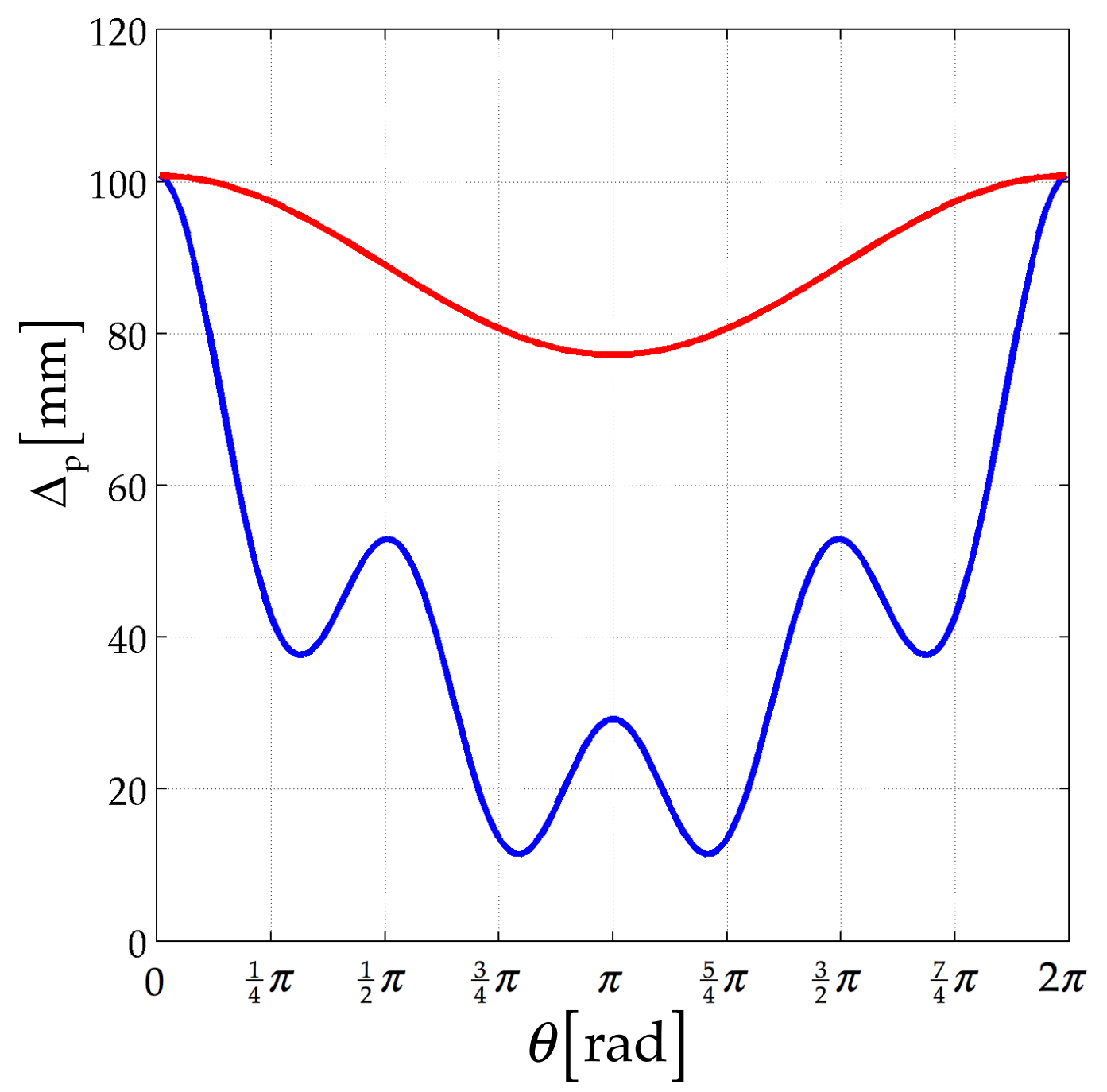

Figure 16: Thickness distribution of the extrudate entering the cooling chamber, $\Delta_{\mathrm{p}}=R_{\mathrm{op}}-R_{i \mathrm{p}}$, (blue) predicted from Eq. (67) [with Eq. (47)] using $P=8.46 \times 10^{5} \mathrm{~Pa} / \mathrm{m}, \eta_{0}=9.6 \mathrm{MPa} \cdot \mathrm{s}, V=1.98 \times 10^{-5} \mathrm{~m} / \mathrm{s}, R_{i}=0.351 \mathrm{~m}$, $R_{o}=0.44 \mathrm{~m}, R_{o \mathrm{p}}=0.44 \mathrm{~m}, \delta=0.0118 \mathrm{~m}, \lambda_{1}=2.38 \mathrm{~s}, \lambda_{2}=\mu_{0}=\mu_{1}=\mu_{2}=v_{1}=v_{2}=0$ versus the die shape (red). 


\section{REFERENCES}

1 Githuku, D.N. and A.J. Giacomin, "A Spectral Element Simulation of Gravitational Flow during Plastic Pipe Extrusion," Journal of Engineering Materials and Technology, 115(4), 433-439 (1993).

2

Githuku, D.N. and A. J. Giacomin, "Elimination of Sag in Plastic Pipe Extrusion," International Polymer Processing, 7(2), 140-143 (1992).

Giacomin, A.J., T. Habinak and S.R. Doshi, "Dies for Pipe Extrusion Which Compensate for Gravity Flow," Proceedings, Polymer Processing Society, Fourth Annual Meeting, Orlando, FL (May 8-11, 1988), p. 4/15.

Giacomin, A.J. and S.R. Doshi, "Analysis of Slump in Plastic Pipe Extrusion," S.P.E. Tech. Paper, XXXIV, Proc. 46th Annual Tech. Conf. \& Exhib., Society of Plastics Engineers, Atlanta, GA (April, 1988), pp. 38-40.

Githuku, D.N., “Simulation of Sag in Plastic Pipe Extrusion,” PhD Thesis, Mechanical Engineering Department, Texas A\&M University, College Station, TX (1992).

Githuku, D.N. and A.J. Giacomin, "Simulation of Slump in Plastic Pipe Extrusion," Journal of Engineering Materials and Technology 114(1), 81-83 (1992).

Pittman, J.F.T. and I.A. Farah, "Production of Pipe with Uniform Wall Thickness: How to Compensate for Gravity Sag," International Polymer Processing, 15(3), 260-267 (2000).

DesLauriers, P.J., M.P. McDaniel, D.C. Rohlfing, R.K. Krishnaswamy, S.J. Secora, E.A. Benham, P.L. Maeger, A.R. Wolfe, A.M. Sukhadia and B.B. Beaulieu, "A Comparative Study of Multimodal vs. Bimodal Polyethylene Pipe Resins for PE-100 Applications," Polymer Engineering $\mathcal{E}$ Science, 45(9), 1203-1213 (2005).

Fischer, P, "Computer-Control on Extrusion Lines," Kunststoffe-German Plastics, 74(7), 362-366 (1984).

Schmalzer, A.M., A.M. Mertz, D.N. Githuku and A.J. Giacomin, “Solidifying Plastic Pipe," Journal of Advanced Engineering (先進工程學 刊), 7(3), 135-143 (2012).

11 Saengow, C., P. Poungthong, C. Kolitawong and A.J. Giacomin, "Plastic Pipe Solidification: Exact Solutions in Cylindrical Coordinates," PRG Report No. 019, Queen's University, Kingston, Canada (2015).

Saengow, C., A.J. Giacomin and C. Kolitawong, "Extruding Plastic Pipe from Eccentric Dies," Journal of Non-Newtonian Fluid Mechanics, 223, 176-199 (2015).

Bird, R.B., R.C. Armstrong and O. Hassager, Dynamics of Polymeric Liquids, Vol. 1, 1st ed., Wiley, New York (1977).

Bird, R.B., R.C. Armstrong and O. Hassager, Dynamics of Polymeric Liquids, Vol. 1, 2nd ed., Wiley, New York (1987). Erratum: In the first printing, the entry for $b_{3}$ in column 3 of Table 6.2-2 " $\eta_{0} \lambda_{1}\left(\lambda_{1}\left(\lambda_{1}-\lambda_{2}\right)\right.$ " should be " $\eta_{0} \lambda_{1}\left(\lambda_{1}-\lambda_{2}\right)$ "

Tadmor, Z. and R.B. Bird, "Rheological Analysis of Stabilizing Forces in Wire Coating Dies," Polymer Engineering E Science, 14(2), 124-136 (1974). 
Tadmor, Z. and C.G. Gogos, Principles of Polymer Processing, WileyInterscience, 2nd ed., Hoboken, NJ (2006).

Kolitawong, C. and A.J. Giacomin, "Dynamic Response of A Shear Stress Transducer in the Sliding Plate Rheometer," Journal of Non-Newtonian Fluid Mechanics, 102(1), 71-96 (2002).

Kolitawong, C., "Local Shear Stress Transduction in Sliding Plate Rheometry," PhD Thesis, University of Wisconsin, Mechanical Engineering Department, University of Wisconsin, Madison, WI (2002).

Kolitawong, C. and A.J. Giacomin, "Axial Flow between Eccentric Cylinders," Polymer-Plastics Technology and Engineering, 40(3), 373-394 (2001). Errata: in Ref. 9 and Table 1., "Buckes" should be "Guckes"; in Ref. 11 and Table 1., "1932" should be "1930"; in Ref. 11, "J. J." should be "J., J.".

Kolitawong, C., A.J. Giacomin and U. Nontakaew, "Viscous Dissipation in Plastic Pipe Extrusion," Polymer Engineering \& Science, 53(10), 2205-2218 (2013).

Moon, P. and D.E. Spencer, Field Theory Handbook: Including Coordinates Systems Differential Equations and Their Solutions, Springer-Verlag, Berling, (1961).

Moon, P. and D.E. Spencer, Field Theory for Engineers, D. Van Nostrand, NJ (1961).

Bird, R.B., W.E. Stewart and E.N. Lightfoot, Transport Phenomena, Revised 2nd ed., Wiley \& Sons, New York (2007).

Walters, K., Rheometry, Wiley, New York (1975).

Tanner, R.I., Engineering Rheology, Clarendon, Oxford (1985).

Carreau, P.J., D.C.R. De Kee and R.P. Chhabra, Rheology of Polymeric System: Principles and Applications, Hanser, Munich (1997).

Agassant, J.-F., P. Avenas, J.-Ph. Sergent and P.J. Carreau, Polymer Processing: Principle and Modeling, Hanser, Munich (1991).

Larson, R.G., Constitutive Equations for Polymer Melts and Solutions, Butterworths, Boston (1988).

Barnes, H.A., J.F. Hutton and K. Walters, An Introduction to Rheology, Elsevier, Amsterdam (1989).

Abdel-Khalik, S.I., O. Hassager and R.B. Bird, "The Goddard Expansion and the Kinetic Theory for Solutions of Rodlike Macromolecules," The Journal of Chemical Physics, 61(10), 4312-4316 (1974).

Bird, R.B., O. Hassager and S.I. Abdel-Khalik, "Co-Rotational Rheological Models and the Goddard Expansion," AIChE Journal, 20(6), 1041-1066 (1974).

Bird, R.B. “A Modification of the Oldroyd Model for Rigid Dumbbell Suspensions with Brownian Motion," Zeitschrift für angewandte Mathematik und Physik (ZAMP), 23(1), 157-159 (1972).

Bird, R.B. and A.J. Giacomin, "Polymer Fluid Dynamics: Continuum and Molecular Approaches," Annual Review of Chemical and Biomolecular Engineering, 7, 479-507 (2016). 
Lumley, J.L., "Applicability of the Oldroyd Constitutive Equation to Flow of Dilute Polymer Solutions," Physics of Fluids (1958-1988), 14(11), 22822284 (1971).

Lumley, J.L., "Erratum: Applicability of the Oldroyd Constitutive Equation to Flow of Dilute Polymer Solutions," Physics of Fluids (1958-1988), 15(11), 2081-2081 (1972).

Bird, R.B., O. Hassager, R.C. Armstrong, C. Curtiss, Dynamics of Polymeric Liquids, Vol. 2, 1st ed., Wiley, New York (1977).

Bird, R.B. and R.C. Armstrong, "Time-Dependent Flows of Dilute Solutions of Rodlike Macromolecules," The Journal of Chemical Physics, 56(7), 36803682 (1972).

Jones, J.R., "Flow of Elastic-Viscous Liquids in Pipes with Cores (PART ONE)," Journal de Mécanique, 3(1), 79-99 (March, 1964). Errata: In Eqs. (20) and (22a), " a const.," should be "a const.,"; In Eq. (15),

" $9 \sigma_{2} \geq \sigma_{1} \geq 0$ " should be " $9 \sigma_{2} \geq \sigma_{1} \geq 1$ "; In Eq. (54), " $\psi_{n}=$ " should be " $\Psi_{n}=$ "; In Eq. (3.5), " $\omega_{i j}=$ " should be " $\omega_{i k}=$ "; In Eq. (16), " $Z$ " $=x+i y "$ should be " $Z^{*}=x-i y$."; The first term on RHS of Eq. (23) " $\gamma_{2}$ " should

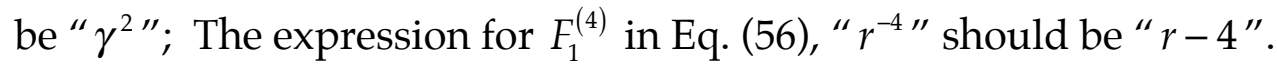
Saengow, C., A.J. Giacomin, P.H. Gilbert and C. Kolitawong, "Reflections on Inflections," Korea-Australia Rheology Journal, 27(4), 267-285 (2015).

Giacomin, A.J., D.N. Githuku, K. Hartwig and K. Stanfill, “Measuring Melt Viscosity Using a Sliding Plate Rheometer with a Shear Stress Transducer," in V.K. Stokes, ed., Plastics and Plastic Composites: Material Properties, Part Performance, and Process Simulation, MDVol. 29, Proc., Symp. on Mechanics of Plastics and Plastic Composites, ASME Winter Annual Meeting, Atlanta, GA, 387-396 (1991).

Doshi, R.S., "A Novel Coextrusion Process for the Manufacture of Short Fiber-Reinforced Thermoplastic Pipe," PhD Thesis, Department of Chemical Engineering, McGill University, Montreal, Canada (1986).

Hade, A.J., A.J. Giacomin, J.C. Slattery and D.N. Githuku, "Postdie Extrusion of Plastic Pipe," Polymer-Plastics Technology and Engineering, 39(1), 23-46 (2000).

Hade, A.J. and A.J. Giacomin, "Power Law Numerical Solution for Post-Die Extrusion of Plastic Pipe," Polymer-Plastics Technology and Engineering, 41(1), 1-17 (2002).

Hade, A.J., "Modeling Free Boundary Flows in Wire Coating and Pipe Extrusion," PhD Thesis, University of Wisconsin, Mechanical Engineering Dept., University of Wisconsin, Madison, WI (2003).

Giacomin, A.J., R.B. Bird, L.M. Johnson and A. W. Mix, "Large-Amplitude Oscillatory Shear Flow from the Corotational Maxwell Model," Journal of Non-Newtonian Fluid Mechanics, 166(19), 1081-1099 (2011).

Saengow, C., A.J. Giacomin and C. Kolitawong, "Exact Analytical Solution for Large-Amplitude Oscillatory Shear Flow," Macromolecular Theory and Simulations, 24(4), 352-392 (2015). 